\title{
The application of trigonal curve theory to the second-order Benjamin-Ono hierarchy
}

Guoliang $\mathrm{He}^{1 *}$ and Lin $\mathrm{He}^{2}$

${ }^{*}$ Correspondence: glhemath@163.com

1 Department of Mathematics and Information Science, Zhengzhou University of Light Industry, Zhengzhou, 450002, China Full list of author information is available at the end of the article

\begin{abstract}
By introducing two sets of Lenard recursion equations, the second-order Benjamin-Ono hierarchy is proposed. In view of the characteristic polynomial of Lax matrix, a trigonal curve of arithmetic genus $m-1$ is deduced. Then the trigonal curve theory is used to derive the explicit algebro-geometric solutions represented in theta functions to the second-order Benjamin-Ono hierarchy with the help of the properties of Baker-Akhiezer function, the meromorphic function and the three kinds of Abel differentials.
\end{abstract}

MSC: 35Q51; 37K10; 14H70; 35C99

Keywords: second-order Benjamin-Ono hierarchy; algebro-geometric solutions; trigonal curve

\section{Introduction}

The principal aim of the present paper concerns the algebro-geometric solutions of the second-order Benjamin-Ono hierarchy with the aid of the theory of trigonal curves [1$3]$. To the best of the authors' knowledge, there have been no results about the algebrogeometric solutions of the second-order Benjamin-Ono equation $[4,5]$

$$
u_{t t}=\alpha\left(u^{2}\right)_{x x}+\beta u_{x x x x}
$$

which is used in the analysis of long waves in shallow water and many other physical applications, where $\alpha$ is a constant controlling nonlinearity and the characteristic speed of the long waves, and $\beta$ is the depth of the fluid, although there are some results about the exact solutions of (1.1), such as the pulse-type and kink-type solutions, periodic solitary wave and double periodic solutions, soliton solutions etc., by using the following methods: the Jacobi elliptic function expansion method, the bilinear method, the extended homoclinic test approach, the homogeneous balance method and the lattice Boltzmann method etc. [6-10].

Before turning to the contents of each section, it seems appropriate to review the existing literature on algebro-geometric solutions, which are of great importance for revealing inherent structure mechanism of solutions and describing the quasi-periodic behavior of nonlinear phenomena. During the last few years, there have been fairly mature techniques to construct algebro-geometric solutions of soliton equations associated with $2 \times 2$ matrix spectral problems, such as the KdV, nonlinear Schrödinger, sine-Gordon, Toda equations

○2014 He and He; licensee Springer. This is an Open Access article distributed under the terms of the Creative Commons Attribution License (http://creativecommons.org/licenses/by/2.0), which permits unrestricted use, distribution, and reproduction in any medium, provided the original work is properly cited. 
and so on [11-15]. Unfortunately, the situation is not so good for soliton equations associated with $3 \times 3$ matrix spectral problems, which are more complicated and more difficult. In [16], a unified framework was proposed to yield all algebro-geometric solutions of the entire Boussinesq hierarchy. Recently, based on the characteristic polynomial of Lax matrix associated with the $3 \times 3$ matrix spectral problems, we have developed the method in [16] to deal with some important soliton equations by introducing the trigonal curves of arithmetic genus $m-1$ and deriving the explicit Riemann theta function representations of the entire hierarchies, such as the modified Boussinesq, the Kaup-Kupershmidt hierarchies and others [17-19].

The present paper is organized as follows. In Section 2, based on two kinds of different Lenard recursion equations, we derive the second-order Benjamin-Ono hierarchy, which relates to a $3 \times 3$ matrix spectral problem. In Section 3, we introduce the Baker-Akhiezer function and the associated meromorphic function. Then the second-order BenjaminOno hierarchy is decomposed into the system of Dubrovin-type ordinary differential equations. In Section 4, the explicit Riemann theta function representations of the BakerAkhiezer function and the meromorphic function, and especially of the solutions to the entire second-order Benjamin-Ono hierarchy are displayed by resorting to the Riemann theta functions, the holomorphic differentials, and the Abel map.

\section{The zero-curvature representation to the second-order Benjamin-Ono hierarchy}

In this section, we shall derive the second-order Benjamin-Ono hierarchy associated with the $3 \times 3$ matrix spectral problem

$$
\psi_{x}=U \psi, \quad \psi=\left(\begin{array}{c}
\psi_{1} \\
\psi_{2} \\
\psi_{3}
\end{array}\right), \quad U=\left(\begin{array}{ccc}
0 & 1 & 0 \\
u & 0 & 1 \\
v+\lambda & u & 0
\end{array}\right),
$$

where $u$ and $v$ are two potentials, and $\lambda$ is a constant spectral parameter. To this end, we introduce two sets of Lenard recursion equations

$$
\begin{aligned}
& K g_{j-1}=J g_{j},\left.\quad g_{j}\right|_{(u, v)=0}=0, j \geq 0, \\
& K \hat{g}_{j-1}=J \hat{g}_{j},\left.\quad \hat{g}_{j}\right|_{(u, v)=0}=0, j \geq 0
\end{aligned}
$$

with two starting points

$$
g_{-1}=(1,0)^{T}, \quad \hat{g}_{-1}=(0,1)^{T},
$$

where the initial conditions mean to identify constants of integration as zero, and two operators are defined as follows:

$$
\begin{array}{lc}
K=\left(\begin{array}{cc}
\partial u+u \partial-\partial^{3} & \partial v+\frac{1}{2} \nu \partial \\
2 v \partial+\partial v & \frac{1}{6} \partial^{5}-\frac{1}{3}\left(\partial^{3} u+u \partial^{3}\right)-\frac{1}{2}\left(\partial^{2} u \partial+\partial u \partial^{2}\right)+u^{2} \partial+\partial u^{2}+\frac{2}{3} u \partial u
\end{array}\right), \\
J=\left(\begin{array}{cc}
0 & -\frac{3}{2} \partial \\
-3 \partial & 0
\end{array}\right) .
\end{array}
$$


Hence $g_{j}$ and $\hat{g}_{j}$ are uniquely determined, for example, the first two members read as

$$
g_{0}=-\frac{1}{3}\left(\begin{array}{c}
v \\
2 u
\end{array}\right), \quad \hat{g}_{0}=\frac{1}{9}\left(\begin{array}{c}
u_{x x}-4 u^{2} \\
-6 v
\end{array}\right)
$$

In order to generate a hierarchy of evolution equations associated with the spectral problem (2.1), we solve the stationary zero-curvature equation

$$
V_{x}-[U, V]=0, \quad V=\left(V_{i j}\right)_{3 \times 3},
$$

which is equivalent to

$$
\begin{aligned}
& V_{11, x}+u V_{12}+(v+\lambda) V_{13}-V_{21}=0, \\
& V_{12, x}+u V_{13}+V_{11}-V_{22}=0, \\
& V_{13, x}-V_{23}+V_{12}=0, \\
& V_{21, x}+u\left(V_{22}-V_{11}\right)+(v+\lambda) V_{23}-V_{31}=0, \\
& V_{22, x}+u\left(V_{23}-V_{12}\right)+V_{21}-V_{32}=0, \\
& V_{23, x}-u V_{13}+V_{22}-V_{33}=0, \\
& V_{31, x}+u\left(V_{32}-V_{21}\right)+(v+\lambda)\left(V_{33}-V_{11}\right)=0, \\
& V_{32, x}+u\left(V_{33}-V_{22}\right)-(v+\lambda) V_{12}+V_{31}=0, \\
& V_{33, x}-u V_{23}-(v+\lambda) V_{13}+V_{32}=0,
\end{aligned}
$$

where each entry $V_{i j}=V_{i j}(a, b)$ is a Laurent expansion in $\lambda$ :

$$
\begin{aligned}
& V_{11}=\frac{1}{3}\left(\frac{1}{2} \partial^{2}-u\right) b-\partial a, \quad V_{12}=a-\frac{1}{2} \partial b, \quad V_{13}=b \\
& V_{21}=\left(\frac{1}{6} \partial^{3}-\frac{1}{3} \partial u-\frac{1}{2} u \partial+v+\lambda\right) b+\left(u-\partial^{2}\right) a, \quad V_{22}=\frac{1}{3}\left(-\partial^{2}+2 u\right) b, \\
& V_{23}=a+\frac{1}{2} \partial b, \quad V_{31}=\left(\frac{1}{6} \partial^{4}-\frac{1}{3} \partial^{2} u-\frac{1}{2} \partial u \partial-\frac{1}{2} u \partial^{2}+u^{2}\right) b+(v+\lambda) a, \\
& V_{32}=\left(-\frac{1}{6} \partial^{3}+\frac{1}{3} \partial u+\frac{1}{2} u \partial+v+\lambda\right) b+\left(u-\partial^{2}\right) a, \\
& V_{33}=\frac{1}{3}\left(\frac{1}{2} \partial^{2}-u\right) b+\partial a, \\
& a=\sum_{j \geq 0} a_{j-1} \lambda^{-j}, \quad b=\sum_{j \geq 0} b_{j-1} \lambda^{-j} .
\end{aligned}
$$

A direct calculation shows that (2.5) and (2.6) imply the Lenard equation

$$
K G=\lambda J G, \quad G=(a, b)^{T} .
$$


Substituting (2.7) into (2.8) and collecting terms with the same powers of $\lambda$, we arrive at the following recursion relation:

$$
K G_{j-1}=J G_{j}, \quad J G_{-1}=0, \quad j \geq 0,
$$

where $G_{j}=\left(a_{j}, b_{j}\right)^{T}$. Since the equation $J G_{-1}=0$ has the general solution

$$
G_{-1}=\alpha_{0} g_{-1}+\beta_{0} \hat{g}_{-1}
$$

then $G_{j}$ can be expressed as

$$
G_{j}=\alpha_{0} g_{j}+\beta_{0} \hat{g}_{j}+\cdots+\alpha_{j} g_{0}+\beta_{j} \hat{g}_{0}+\alpha_{j+1} g_{-1}+\beta_{j+1} \hat{g}_{-1}, \quad j \geq 0,
$$

where $\alpha_{j}$ and $\beta_{j}$ are arbitrary constants.

Let $\psi$ satisfy the spectral problem (2.1) and its auxiliary problem

$$
\psi_{t_{r}}=\widetilde{V}^{(r)} \psi, \quad \widetilde{V}^{(r)}=\left(\widetilde{V}_{i j}^{(r)}\right)_{3 \times 3},
$$

where each entry $\widetilde{V}_{i j}^{(r)}=\widetilde{V}_{i j}\left(\tilde{a}^{(r)}, \tilde{b}^{(r)}\right)$,

$$
\tilde{a}^{(r)}=\sum_{j=0}^{r} \tilde{a}_{j-1} \lambda^{r-j}, \quad \tilde{b}^{(r)}=\sum_{j=0}^{r} \tilde{a}_{j-1} \lambda^{r-j}
$$

with

$$
\widetilde{G}_{j}=\left(\tilde{a}_{j}, \tilde{b}_{j}\right)^{T}=\tilde{\alpha}_{0} g_{j}+\tilde{\beta}_{0} \hat{g}_{j}+\cdots+\tilde{\alpha}_{j} g_{0}+\tilde{\beta}_{j} \hat{g}_{0}+\tilde{\alpha}_{j+1} g_{-1}+\tilde{\beta}_{j+1} \hat{g}_{-1}, \quad j \geq-1 .
$$

Then the compatibility condition of (2.1) and (2.12) yields the zero-curvature equation, $U_{t_{r}}-\widetilde{V}_{x}^{(r)}+\left[U, \widetilde{V}^{(r)}\right]=0$, which is equivalent to the hierarchy of nonlinear evolution equations

$$
\left(u_{t_{r}}, v_{t_{r}}\right)^{T}=\widetilde{X}_{r}, \quad r \geq 0,
$$

where the vector fields $\widetilde{X}_{j}=\widetilde{X}_{j}\left(u, v ; \underline{\tilde{\alpha}}^{(j)}, \underline{\tilde{\beta}}^{(j)}\right)=K \widetilde{G}_{j-1}=J \widetilde{G}_{j}$, and $\underline{\tilde{\alpha}}^{(j)}=\left(\tilde{\alpha}_{0}, \ldots, \tilde{\alpha}_{j}\right), \underline{\tilde{\beta}}^{(j)}=$ $\left(\tilde{\beta}_{0}, \ldots, \tilde{\beta}_{j}\right)$. The first nontrivial member in the hierarchy $(2.13)$ is as follows:

$$
\begin{aligned}
& u_{t_{0}}=\tilde{\alpha}_{0} u_{x}+\tilde{\beta}_{0} v_{x}, \\
& v_{t_{0}}=\tilde{\alpha}_{0} v_{x}-\frac{1}{3} \tilde{\beta}_{0}\left(u_{x x x}-8 u u_{x}\right) .
\end{aligned}
$$

For $\tilde{\alpha}_{0}=0, \tilde{\beta}_{0}=1\left(t_{0}=t\right)$, equation (2.14) is reduced to the second-order Benjamin-Ono equation by canceling the variable $v$

$$
u_{t t}=\frac{4}{3}\left(u^{2}\right)_{x x}-\frac{1}{3} u_{x x x x} .
$$


The second one in the hierarchy (2.13) (as $\left.\tilde{\alpha}_{1}=0, \tilde{\beta}_{1}=0\right)$ can be written as

$$
\begin{aligned}
u_{t_{1}}= & \frac{1}{3} \tilde{\alpha}_{0}\left(v_{x x}-4 u v\right)_{x}-\frac{1}{54} \tilde{\beta}_{0}\left(6 u_{x x x x}-60 u u_{x x}-45 u_{x}^{2}+40 u^{3}+45 v^{2}\right)_{x} \\
v_{t_{1}}= & -\frac{1}{27} \tilde{\alpha}_{0}\left(3 u_{x x x x}-36 u u_{x x}-18 u_{x}^{2}+32 u^{3}+18 v^{2}\right)_{x} \\
& -\frac{1}{9} \tilde{\beta}_{0}\left(v_{x x x x}-5 u_{x x} v-10 u v_{x x}-5 u_{x} v_{x}+20 u^{2} v\right)_{x}
\end{aligned}
$$

For $\tilde{\alpha}_{0}=0, \tilde{\beta}_{0}=-9\left(t_{1}=t\right)$, equation (2.16) is reduced to a 5 -order coupled equation

$$
\begin{aligned}
& u_{t}=u_{x x x x x}-\left(10 u u_{x x}+9 u_{x}^{2}-9 v^{2}-\frac{20}{3} u^{3}\right)_{x} \\
& v_{t}=v_{x x x x x}-\left(5 u_{x x} v+10 u v_{x x}+5 u_{x} v_{x}-20 u^{2} v\right)_{x} .
\end{aligned}
$$

\section{The meromorphic function and Dubrovin-type equations}

In this section, we shall consider the Baker-Akhiezer function and the associated meromorphic function. By introducing the elliptic kind coordinates, we decompose the secondorder Benjamin-Ono equation into the system of Dubrovin-type differential equations.

We first introduce the Baker-Akhiezer function $\psi\left(P, x, x_{0}, t_{r}, t_{0, r}\right)$ by

$$
\begin{aligned}
& \psi_{x}\left(P, x, x_{0}, t_{r}, t_{0, r}\right)=U\left(u\left(x, t_{r}\right), v\left(x, t_{r}\right) ; \lambda(P)\right) \psi\left(P, x, x_{0}, t_{r}, t_{0, r}\right), \\
& \psi_{t_{r}}\left(P, x, x_{0}, t_{r}, t_{0, r}\right)=\widetilde{V}^{(r)}\left(u\left(x, t_{r}\right), v\left(x, t_{r}\right) ; \lambda(P)\right) \psi\left(P, x, x_{0}, t_{r}, t_{0, r}\right), \\
& V^{(n)}\left(u\left(x, t_{r}\right), v\left(x, t_{r}\right) ; \lambda(P)\right) \psi\left(P, x, x_{0}, t_{r}, t_{0, r}\right)=y(P) \psi\left(P, x, x_{0}, t_{r}, t_{0, r}\right), \\
& \psi_{1}\left(P, x_{0}, x_{0}, t_{0, r}, t_{0, r}\right)=1,
\end{aligned}
$$

where $V^{(n)}=\left(\lambda^{n} V\right)_{+}=\left(V_{i j}^{(n)}\right)_{3 \times 3}$ and $V_{i j}^{(n)}=V_{i j}\left(a^{(n)}, b^{(n)}\right)$,

$$
a^{(n)}=\sum_{j=0}^{n} a_{j-1} \lambda^{n-j}, \quad b^{(n)}=\sum_{j=0}^{n} b_{j-1} \lambda^{n-j}
$$

with $a_{j}, b_{j}$ determined by (2.11). The compatibility conditions of the first three expressions in (3.1) yield that

$$
\begin{aligned}
& U_{t_{r}}-\widetilde{V}_{x}^{(r)}+\left[U, \widetilde{V}^{(r)}\right]=0, \\
& -V_{x}^{(n)}+\left[U, V^{(n)}\right]=0, \\
& -V_{t_{r}}^{(n)}+\left[\widetilde{V}^{(r)}, V^{(n)}\right]=0 .
\end{aligned}
$$

Through a direct calculation we can show that $y I-V^{(n)}$ satisfies equations (3.3) and (3.4). So $\mathcal{F}_{m}(\lambda, y)=\operatorname{det}\left(y I-V^{(n)}\right)$ is an independent constant of the variables $x$ and $t_{r}$, from which we can define a trigonal curve $\mathcal{K}_{m-1}: \mathcal{F}_{m}(\lambda, y)=0$ with the expansion

$$
\operatorname{det}\left(y I-V^{(n)}\right)=y^{3}+y S_{m}(\lambda)-T_{m}(\lambda)=0,
$$


where

$$
S_{m}=\sum_{1 \leq i<j \leq 3}\left|\begin{array}{cc}
V_{i i}^{(n)} & V_{i j}^{(n)} \\
V_{j i}^{(n)} & V_{j j}^{(n)}
\end{array}\right|, \quad T_{m}=\left|\begin{array}{ccc}
V_{11}^{(n)} & V_{12}^{(n)} & V_{13}^{(n)} \\
V_{21}^{(n)} & V_{22}^{(n)} & V_{23}^{(n)} \\
V_{31}^{(n)} & V_{32}^{(n)} & V_{33}^{(n)}
\end{array}\right| .
$$

Immediately, from (2.10) if we choose $\beta_{0}=1, \alpha_{0}$ an arbitrary constant or $\beta_{0}=0, \alpha_{0}=1$, we shall know that the corresponding values of $m$ in (3.5) are $3 n+2$ or $3 n+1$, respectively. For the convenience, the compactification of the curve $\mathcal{K}_{m-1}$ is denoted by the same symbol $\mathcal{K}_{m-1}$. Thus $\mathcal{K}_{m-1}$ becomes a three-sheeted Riemann surface of arithmetic genus $m-1$ when it is nonsingular or smooth.

Next we shall introduce the meromorphic function $\phi_{1}\left(P, x, t_{r}\right)$, which is closely related to $\psi\left(P, x, x_{0}, t_{r}, t_{0, r}\right)$, by

$$
\phi_{1}\left(P, x, t_{r}\right)=\frac{\partial_{x} \psi_{1}\left(P, x, x_{0}, t_{r}, t_{0, r}\right)}{\psi_{1}\left(P, x, x_{0}, t_{r}, t_{0, r}\right)}, \quad P \in \mathcal{K}_{m-1}, x \in \mathbb{C}
$$

which implies from (3.1) that

$$
\begin{aligned}
\phi_{1}\left(P, x, t_{r}\right) & =\frac{\varepsilon(m) F_{m}\left(\lambda, x, t_{r}\right)}{y^{2} V_{23}^{(n)}\left(\lambda, x, t_{r}\right)-y C_{m}\left(\lambda, x, t_{r}\right)+D_{m}\left(\lambda, x, t_{r}\right)} \\
& =\frac{y^{2} V_{13}^{(n)}\left(\lambda, x, t_{r}\right)-y A_{m}\left(\lambda, x, t_{r}\right)+B_{m}\left(\lambda, x, t_{r}\right)}{-\varepsilon(m) E_{m-1}\left(\lambda, x, t_{r}\right)} \\
& =\frac{y V_{23}^{(n)}\left(\lambda, x, t_{r}\right)+C_{m}\left(\lambda, x, t_{r}\right)}{y V_{13}^{(n)}\left(\lambda, x, t_{r}\right)+A_{m}\left(\lambda, x, t_{r}\right)}
\end{aligned}
$$

where $P=(\lambda, y) \in \mathcal{K}_{m-1},\left(x, t_{r}\right) \in \mathbb{C}^{2}$,

$$
\begin{aligned}
& A_{m}=V_{12}^{(n)} V_{23}^{(n)}-V_{13}^{(n)} V_{22}^{(n)}, \\
& B_{m}=V_{13}^{(n)}\left(V_{11}^{(n)} V_{33}^{(n)}-V_{13}^{(n)} V_{31}^{(n)}\right)+V_{12}^{(n)}\left(V_{11}^{(n)} V_{23}^{(n)}-V_{13}^{(n)} V_{21}^{(n)}\right), \\
& C_{m}=V_{13}^{(n)} V_{21}^{(n)}-V_{11}^{(n)} V_{23}^{(n)}, \\
& D_{m}=V_{23}^{(n)}\left(V_{22}^{(n)} V_{33}^{(n)}-V_{23}^{(n)} V_{32}^{(n)}\right)+V_{21}^{(n)}\left(V_{13}^{(n)} V_{22}^{(n)}-V_{12}^{(n)} V_{23}^{(n)}\right), \\
& E_{m-1}=-\varepsilon(m)\left[V_{13}^{(n)}\left(V_{13}^{(n)} V_{32}^{(n)}-V_{12}^{(n)} V_{33}^{(n)}\right)+V_{12}^{(n)}\left(V_{13}^{(n)} V_{22}^{(n)}-V_{12}^{(n)} V_{23}^{(n)}\right)\right], \\
& F_{m}=\varepsilon(m)\left[V_{23}^{(n)}\left(V_{23}^{(n)} V_{31}^{(n)}-V_{21}^{(n)} V_{33}^{(n)}\right)+V_{21}^{(n)}\left(V_{11}^{(n)} V_{23}^{(n)}-V_{13}^{(n)} V_{21}^{(n)}\right)\right],
\end{aligned}
$$

and

$$
\varepsilon(m)= \begin{cases}-1 & \text { if } m=3 n+2 \\ 1 & \text { if } m=3 n+1\end{cases}
$$

which is introduced to ensure that $E_{m-1}, F_{m}$ are both monic polynomials. It is easy to see that there exist various interrelationships between polynomials $A_{m}, B_{m}, C_{m}, D_{m}, E_{m-1}, F_{m}$ 
and $S_{m}, T_{m}$, some of which are summarized as follows:

$$
\begin{aligned}
& \varepsilon(m) V_{13}^{(n)} F_{m}=V_{23}^{(n)} D_{m}-S_{m}\left(V_{23}^{(n)}\right)^{2}-C_{m}^{2}, \\
& \varepsilon(m) A_{m} F_{m}=T_{m}\left(V_{23}^{(n)}\right)^{2}+C_{m} D_{m}, \\
& \varepsilon(m) V_{23}^{(n)} E_{m-1}=S_{m}\left(V_{13}^{(n)}\right)^{2}-V_{13}^{(n)} B_{m}+A_{m}^{2}, \\
& -\varepsilon(m) C_{m} E_{m-1}=T_{m}\left(V_{13}^{(n)}\right)^{2}+A_{m} B_{m}, \\
& V_{23}^{(n)} B_{m}+V_{13}^{(n)} D_{m}-V_{13}^{(n)} V_{23}^{(n)} S_{m}+A_{m} C_{m}=0, \\
& V_{13}^{(n)} V_{23}^{(n)} T_{m}+V_{23}^{(n)} A_{m} S_{m}+V_{13}^{(n)} C_{m} S_{m}-B_{m} C_{m}-A_{m} D_{m}=0, \\
& V_{23}^{(n)} A_{m} T_{m}+V_{13}^{(n)} C_{m} T_{m}-E_{m-1} F_{m}-B_{m} D_{m}=0, \\
& \varepsilon(m) E_{m-1, x}=2 S_{m} V_{13}^{(n)}-3 B_{m}, \\
& V_{23}^{(n)} F_{m, x}=-3 V_{22}^{(n)} F_{m}+\varepsilon(m)\left(V_{21}^{(n)}-u V_{23}^{(n)}\right)\left(2 V_{23}^{(n)} S_{m}-3 D_{m}\right) .
\end{aligned}
$$

For displaying the properties of $\phi_{1}\left(P, x, t_{r}\right)$ exactly, we introduce the holomorphic map $*$, changing sheets, as

$$
\begin{aligned}
& *:\left\{\begin{array}{l}
\mathcal{K}_{m-1} \rightarrow \mathcal{K}_{m-1}, \\
P=\left(\lambda, y_{i}(\lambda)\right) \rightarrow P^{*}=\left(\lambda, y_{i+1(\bmod 3)}(\lambda)\right), \quad i=0,1,2,
\end{array}\right. \\
& P^{* *}:=\left(P^{*}\right)^{*}, \quad \text { etc. },
\end{aligned}
$$

where $y_{i}(\lambda), i=0,1,2$, denote the three branches of $y(P)$ satisfying $\mathcal{F}_{m}(\lambda, y)=0$. Then it is easy to show the properties of $\phi_{1}\left(P, x, t_{r}\right)$ immediately:

$$
\begin{aligned}
& \phi_{1, x x}\left(P, x, t_{r}\right)+3 \phi_{1}\left(P, x, t_{r}\right) \phi_{1, x}\left(P, x, t_{r}\right)+\phi_{1}^{3}\left(P, x, t_{r}\right)-2 u\left(x, t_{r}\right) \phi_{1}\left(P, x, t_{r}\right) \\
& =u_{x}\left(x, t_{r}\right)+v\left(x, t_{r}\right)+\lambda, \\
& \phi_{1, t_{r}}\left(P, x, t_{r}\right)=\partial_{x}\left[\widetilde{V}_{11}^{(r)}\left(\lambda, x, t_{r}\right)+\widetilde{V}_{12}^{(r)}\left(\lambda, x, t_{r}\right) \phi_{1}\left(P, x, t_{r}\right)\right. \\
& \left.+\widetilde{V}_{13}^{(r)}\left(\lambda, x, t_{r}\right)\left(\phi_{1, x}\left(P, x, t_{r}\right)+\phi_{1}^{2}\left(P, x, t_{r}\right)-u\left(x, t_{r}\right)\right)\right], \\
& \phi_{1}\left(P, x, t_{r}\right) \phi_{1}\left(P^{*}, x, t_{r}\right) \phi_{1}\left(P^{* *}, x, t_{r}\right)=\frac{F_{m}\left(\lambda, x, t_{r}\right)}{E_{m-1}\left(\lambda, x, t_{r}\right)}, \\
& \phi_{1}\left(P, x, t_{r}\right)+\phi_{1}\left(P^{*}, x, t_{r}\right)+\phi_{1}\left(P^{* *}, x, t_{r}\right)=\frac{E_{m-1, x}\left(\lambda, x, t_{r}\right)}{E_{m-1}\left(\lambda, x, t_{r}\right)}, \\
& y(P) \phi_{1}\left(P, x, t_{r}\right)+y\left(P^{*}\right) \phi_{1}\left(P^{*}, x, t_{r}\right)+y\left(P^{* *}\right) \phi_{1}\left(P^{* *}, x, t_{r}\right) \\
& =\frac{3 T_{m}(\lambda) V_{32}^{(n)}\left(\lambda, x, t_{r}\right)+2 S_{m}(\lambda) A_{m}\left(\lambda, x, t_{r}\right)}{-\varepsilon(m) E_{m-1}\left(\lambda, x, t_{r}\right)}, \\
& \frac{1}{\phi_{1}\left(P, x, t_{r}\right)}+\frac{1}{\phi_{1}\left(P^{*}, x, t_{r}\right)}+\frac{1}{\phi_{1}\left(P^{* *}, x, t_{r}\right)} \\
& =\frac{-3 V_{22}^{(n)}\left(\lambda, x, t_{r}\right)}{V_{21}^{(n)}\left(\lambda, x, t_{r}\right)-u\left(x, t_{r}\right) V_{23}^{(n)}\left(\lambda, x, t_{r}\right)} \\
& -\frac{V_{23}^{(n)}\left(\lambda, x, t_{r}\right)}{V_{21}^{(n)}\left(\lambda, x, t_{r}\right)-u\left(x, t_{r}\right) V_{23}^{(n)}\left(\lambda, x, t_{r}\right)} \frac{F_{m, x}\left(\lambda, x, t_{r}\right)}{F_{m}\left(\lambda, x, t_{r}\right)} .
\end{aligned}
$$


After tedious calculations, we have the following lemma.

Lemma 3.1 Assume (3.1), (3.2), and let $\left(\lambda, x, x_{0}, t_{r}, t_{0, r}\right) \in \mathbb{C}^{5}$. Then

$$
\begin{aligned}
E_{m-1, t_{r}}\left(\lambda, x, t_{r}\right) & =E_{m-1, x}\left(\widetilde{V}_{12}^{(r)}-\frac{\widetilde{V}_{13}^{(r)}}{V_{13}^{(n)}} V_{12}^{(n)}\right)+3 E_{m-1}\left(\widetilde{V}_{11}^{(r)}-\frac{\widetilde{V}_{13}^{(r)}}{V_{13}^{(n)}} V_{11}^{(n)}\right) \\
F_{m, t_{r}}\left(\lambda, x, t_{r}\right)= & F_{m, x}\left(\widetilde{V}_{23}^{(r)}-\frac{\widetilde{V}_{21}^{(r)}-u \widetilde{V}_{23}^{(r)}}{V_{21}^{(n)}-u V_{23}^{(n)}} V_{23}^{(n)}\right) \\
& +3 F_{m}\left(\widetilde{V}_{22}^{(r)}-\frac{\widetilde{V}_{21}^{(r)}-u \widetilde{V}_{23}^{(r)}}{V_{21}^{(n)}-u V_{23}^{(n)}} V_{22}^{(n)}\right) .
\end{aligned}
$$

Moreover, by institute of (3.2), (3.6), (3.16), and (3.19), we arrive at the properties of $\psi_{1}\left(P, x, x_{0}, t_{r}, t_{0, r}\right)$ immediately.

Lemma 3.2 Assume (3.1), (3.6), $P=(\lambda, y(P)) \in \mathcal{K}_{m-1} \backslash\left\{P_{\infty}\right\}$, and let $\left(\lambda, x, x_{0}, t_{r}, t_{0, r}\right) \in \mathbb{C}^{5}$. Then

$$
\begin{aligned}
& \frac{\psi_{1, t_{r}}\left(P, x, x_{0}, t_{r}, t_{0, r}\right)}{\psi_{1}\left(P, x, x_{0}, t_{r}, t_{0, r}\right)}=\widetilde{V}_{13}^{(r)}\left(\lambda, x, t_{r}\right)\left[\phi_{1, x}\left(P, x, t_{r}\right)+\phi_{1}^{2}\left(P, x, t_{r}\right)-u\left(x, t_{r}\right)\right] \\
& +\widetilde{V}_{12}^{(r)}\left(\lambda, x, t_{r}\right) \phi_{1}\left(P, x, t_{r}\right)+\widetilde{V}_{11}^{(r)}\left(\lambda, x, t_{r}\right) \\
& \psi_{1}\left(P, x, x_{0}, t_{r}, t_{0, r}\right) \psi_{1}\left(P^{*}, x, x_{0}, t_{r}, t_{0, r}\right) \psi_{1}\left(P^{* *}, x, x_{0}, t_{r}, t_{0, r}\right)=\frac{E_{m-1}\left(\lambda, x, t_{r}\right)}{E_{m-1}\left(\lambda, x_{0}, t_{0, r}\right)}, \\
& \psi_{1, x}\left(P, x, x_{0}, t_{r}, t_{0, r}\right) \psi_{1, x}\left(P^{*}, x, x_{0}, t_{r}, t_{0, r}\right) \psi_{1, x}\left(P^{* *}, x, x_{0}, t_{r}, t_{0, r}\right)=\frac{F_{m}\left(\lambda, x, t_{r}\right)}{E_{m-1}\left(\lambda, x_{0}, t_{0, r}\right)}, \\
& \psi_{1}\left(P, x, x_{0}, t_{r}, t_{0, r}\right) \\
& =\exp \left(\int_{x_{0}}^{x} \phi_{1}\left(P, x^{\prime}, t_{r}\right) d x^{\prime}\right. \\
& +\int_{t_{0, r}}^{t_{r}}\left[\widetilde{V}_{13}^{(r)}\left(\lambda, x_{0}, t^{\prime}\right)\left(\frac{y(P)-V_{11}^{(n)}\left(\lambda, x_{0}, t^{\prime}\right)}{V_{13}^{(n)}\left(\lambda, x_{0}, t^{\prime}\right)}-\frac{V_{12}^{(n)}\left(\lambda, x_{0}, t^{\prime}\right)}{V_{13}^{(n)}\left(\lambda, x_{0}, t^{\prime}\right)} \phi_{1}\left(P, x_{0}, t^{\prime}\right)\right)\right. \\
& \left.\left.+\widetilde{V}_{12}^{(r)}\left(\lambda, x_{0}, t^{\prime}\right) \phi_{1}\left(P, x_{0}, t^{\prime}\right)+\widetilde{V}_{11}^{(r)}\left(\lambda, x_{0}, t^{\prime}\right)\right] d t^{\prime}\right) \\
& \psi_{1}\left(P, x, x_{0}, t_{r}, t_{0, r}\right) \\
& =\left[\frac{E_{m-1}\left(\lambda, x, t_{r}\right)}{E_{m-1}\left(\lambda, x_{0}, t_{0, r}\right)}\right]^{1 / 3} \\
& \times \exp \left(\int_{x_{0}}^{x} \frac{y(P)^{2} V_{13}^{(n)}\left(\lambda, x^{\prime}, t_{r}\right)-y(P) A_{m}\left(\lambda, x^{\prime}, t_{r}\right)+\frac{2}{3} S_{m}(\lambda) V_{13}^{(n)}\left(\lambda, x^{\prime}, t_{r}\right)}{-\varepsilon(m) E_{m-1}\left(\lambda, x^{\prime}, t_{r}\right)} d x^{\prime}\right. \\
& +\int_{t_{0, r}}^{t_{r}}\left[\frac{y(P)^{2} V_{13}^{(n)}\left(\lambda, x_{0}, t^{\prime}\right)-y(P) A_{m}\left(\lambda, x_{0}, t^{\prime}\right)+\frac{2}{3} S_{m}(\lambda) V_{13}^{(n)}\left(\lambda, x_{0}, t^{\prime}\right)}{-\varepsilon(m) E_{m-1}\left(\lambda, x_{0}, t^{\prime}\right)}\right. \\
& \times\left(\tilde{V}_{12}^{(r)}\left(\lambda, x_{0}, t^{\prime}\right)-\frac{\tilde{V}_{13}^{(r)}\left(\lambda, x_{0}, t^{\prime}\right)}{V_{13}^{(n)}\left(\lambda, x_{0}, t^{\prime}\right)} V_{12}^{(n)}\left(\lambda, x_{0}, t^{\prime}\right)\right) \\
& \left.\left.+y(P) \frac{\tilde{V}_{13}^{(r)}\left(\lambda, x_{0}, t^{\prime}\right)}{V_{13}^{(n)}\left(\lambda, x_{0}, t^{\prime}\right)}\right] d t^{\prime}\right)
\end{aligned}
$$


By inspection of (3.9), one shall know that $E_{m-1}$ and $F_{m}$ are both monic polynomials with respect to $\lambda$ of degree $m-1$ and $m$, respectively. Hence we may decompose them into

$$
\begin{aligned}
& E_{m-1}\left(\lambda, x, t_{r}\right)=\prod_{j=1}^{m-1}\left(\lambda-\mu_{j}\left(x, t_{r}\right)\right), \\
& F_{m}\left(\lambda, x, t_{r}\right)=\prod_{l=0}^{m-1}\left(\lambda-v_{l}\left(x, t_{r}\right)\right) .
\end{aligned}
$$

Define

$$
\begin{aligned}
& \hat{\mu}_{j}\left(x, t_{r}\right)=\left(\mu_{j}\left(x, t_{r}\right), y\left(\hat{\mu}_{j}\left(x, t_{r}\right)\right)\right)=\left(\mu_{j}\left(x, t_{r}\right),-\frac{A_{m}\left(\mu_{j}\left(x, t_{r}\right), x, t_{r}\right)}{V_{13}^{(n)}\left(\mu_{j}\left(x, t_{r}\right), x, t_{r}\right)}\right) \in \mathcal{K}_{m-1}, \\
& 1 \leq j \leq m-1,\left(x, t_{r}\right) \in \mathbb{C}^{2}, \\
& \hat{v}_{l}\left(x, t_{r}\right)=\left(v_{l}\left(x, t_{r}\right), y\left(\hat{v}_{l}\left(x, t_{r}\right)\right)\right)=\left(v_{l}\left(x, t_{r}\right),-\frac{C_{m}\left(v_{l}\left(x, t_{r}\right), x, t_{r}\right)}{V_{23}^{(n)}\left(v_{l}\left(x, t_{r}\right), x, t_{r}\right)}\right) \in \mathcal{K}_{m-1}, \\
& 0 \leq l \leq m-1,\left(x, t_{r}\right) \in \mathbb{C}^{2} .
\end{aligned}
$$

The dynamics of the zeros $\mu_{j}\left(x, t_{r}\right)$ and $v_{l}\left(x, t_{r}\right)$ of $E_{m-1}\left(\lambda, x, t_{r}\right)$ and $F_{m}\left(\lambda, x, t_{r}\right)$ are then described in terms of Dubrovin-type equations as follows.

Lemma 3.3 (i) Suppose that the zeros $\mu_{j}\left(x, t_{r}\right)_{j=1, \ldots, m-1}$ of $E_{m-1}\left(P, x, t_{r}\right)$ remain distinct for $\left(x, t_{r}\right) \in \Omega_{\mu}$, where $\Omega_{\mu} \subseteq \mathbb{C}^{2}$ is open and connected. Then $\mu_{j}\left(x, t_{r}\right)_{j=1, \ldots, m-1}$ satisfy the system of differential equations

$$
\begin{aligned}
& \mu_{j, x}\left(x, t_{r}\right)= \frac{\varepsilon(m) V_{13}^{(n)}\left(\mu_{j}\left(x, t_{r}\right), x, t_{r}\right)\left[3 y^{2}\left(\hat{\mu}_{j}\left(x, t_{r}\right)\right)+S_{m}\left(\mu_{j}\left(x, t_{r}\right)\right)\right]}{\prod_{\substack{k=1 \\
k \neq j}}^{m-1}\left(\mu_{j}\left(x, t_{r}\right)-\mu_{k}\left(x, t_{r}\right)\right)}, \\
& 1 \leq j \leq m-1, \\
& \mu_{j, t_{r}}\left(x, t_{r}\right)=\left[V_{13}^{(n)}\left(\mu_{j}\left(x, t_{r}\right), x, t_{r}\right) \widetilde{V}_{12}^{(r)}\left(\mu_{j}\left(x, t_{r}\right), x, t_{r}\right)\right. \\
&\left.-\widetilde{V}_{13}^{(r)}\left(\mu_{j}\left(x, t_{r}\right), x, t_{r}\right) V_{12}^{(n)}\left(\mu_{j}\left(x, t_{r}\right), x, t_{r}\right)\right] \\
& \times \frac{\varepsilon(m)\left[3 y^{2}\left(\hat{\mu}_{j}\left(x, t_{r}\right)\right)+S_{m}\left(\mu_{j}\left(x, t_{r}\right)\right)\right]}{\prod_{\substack{k=1 \\
k \neq j}}^{m-1}\left(\mu_{j}\left(x, t_{r}\right)-\mu_{k}\left(x, t_{r}\right)\right)}, 1 \leq j \leq m-1 .
\end{aligned}
$$

(ii) Suppose that the zeros $v_{l}\left(x, t_{r}\right)_{l=0, \ldots, m-1}$ of $F_{m}\left(P, x, t_{r}\right)$ remain distinct for $\left(x, t_{r}\right) \in \Omega_{v}$, where $\Omega_{v} \subseteq \mathbb{C}^{2}$ is open and connected. Then $v_{l}\left(x, t_{r}\right)_{l=0, \ldots, m-1}$ satisfy the system of differential equations

$$
\begin{aligned}
& v_{l, x}\left(x, t_{r}\right) \\
& =\frac{\varepsilon(m)\left[V_{21}^{(n)}\left(v_{l}\left(x, t_{r}\right), x, t_{r}\right)-u V_{23}^{(n)}\left(v_{l}\left(x, t_{r}\right), x, t_{r}\right)\right]\left[3 y^{2}\left(\hat{v}_{l}\left(x, t_{r}\right)\right)+S_{m}\left(v_{l}\left(x, t_{r}\right)\right)\right]}{\prod_{\substack{k=0 \\
k \neq l}}^{m-1}\left(v_{l}\left(x, t_{r}\right)-v_{k}\left(x, t_{r}\right)\right)}, \\
& 0 \leq l \leq m-1,
\end{aligned}
$$




$$
\begin{aligned}
v_{l, t_{r}}\left(x, t_{r}\right)= & {\left[\left(V_{21}^{(n)}\left(v_{l}\left(x, t_{r}\right), x, t_{r}\right)-u V_{23}^{(n)}\left(v_{l}\left(x, t_{r}\right), x, t_{r}\right)\right) \widetilde{V}_{23}^{(r)}\left(v_{l}\left(x, t_{r}\right), x, t_{r}\right)\right.} \\
& \left.-\left(\widetilde{V}_{21}^{(r)}\left(v_{l}\left(x, t_{r}\right), x, t_{r}\right)-u \widetilde{V}_{23}^{(r)}\left(v_{l}\left(x, t_{r}\right), x, t_{r}\right)\right) V_{23}^{(n)}\left(v_{l}\left(x, t_{r}\right), x, t_{r}\right)\right] \\
& \times \frac{\varepsilon(m)\left[3 y^{2}\left(\hat{v}_{l}\left(x, t_{r}\right)\right)+S_{m}\left(v_{l}\left(x, t_{r}\right)\right)\right]}{\prod_{\substack{k=0 \\
k \neq l}}^{m-1}\left(v_{l}\left(x, t_{r}\right)-v_{k}\left(x, t_{r}\right)\right)}, \quad 0 \leq l \leq m-1 .
\end{aligned}
$$

Proof Using (3.10), we have $\left(\lambda=\mu_{j}\left(x, t_{r}\right)\right)$

$$
\begin{aligned}
& S_{m}\left(\mu_{j}\left(x, t_{r}\right)\right)\left(V_{13}^{(n)}\left(\mu_{j}\left(x, t_{r}\right), x, t_{r}\right)\right)^{2}-B_{m}\left(\mu_{j}\left(x, t_{r}\right), x, t_{r}\right) V_{13}^{(n)}\left(\mu_{j}\left(x, t_{r}\right), x, t_{r}\right) \\
& \quad+A_{m}^{2}\left(\mu_{j}\left(x, t_{r}\right), x, t_{r}\right)=0,
\end{aligned}
$$

that is,

$$
\begin{aligned}
B_{m}\left(\mu_{j}\left(x, t_{r}\right), x, t_{r}\right) & =S_{m}\left(\mu_{j}\left(x, t_{r}\right)\right) V_{13}^{(n)}\left(\mu_{j}\left(x, t_{r}\right), x, t_{r}\right)+\frac{A_{m}^{2}\left(\mu_{j}\left(x, t_{r}\right), x, t_{r}\right)}{V_{13}^{(n)}\left(\mu_{j}\left(x, t_{r}\right), x, t_{r}\right)} \\
& =\left[S_{m}\left(\mu_{j}\left(x, t_{r}\right)\right)+y^{2}\left(\hat{\mu}_{j}\left(x, t_{r}\right)\right)\right] V_{13}^{(n)}\left(\mu_{j}\left(x, t_{r}\right), x, t_{r}\right) .
\end{aligned}
$$

After substituting $B_{m}$ into (3.12), we get

$$
\begin{aligned}
& \varepsilon(m) E_{m-1, x}\left(\mu_{j}\left(x, t_{r}\right), x, t_{r}\right) \\
& \quad=-V_{13}^{(n)}\left(\mu_{j}\left(x, t_{r}\right), x, t_{r}\right)\left[3 y^{2}\left(\hat{\mu}_{j}\left(x, t_{r}\right)\right)+S_{m}\left(\mu_{j}\left(x, t_{r}\right)\right)\right] .
\end{aligned}
$$

On the other hand, derivatives of the expression in (3.25) with respect to $x$ and $t_{r}$ respectively, are

$$
\begin{aligned}
& E_{m-1, x}\left(\mu_{j}\left(x, t_{r}\right), x, t_{r}\right)=-\mu_{j, x}\left(x, t_{r}\right) \prod_{\substack{k=1 \\
k \neq j}}^{m-1}\left(\mu_{j}\left(x, t_{r}\right)-\mu_{k}\left(x, t_{r}\right)\right), \\
& E_{m-1, t_{r}}\left(\mu_{j}\left(x, t_{r}\right), x, t_{r}\right)=-\mu_{j, t_{r}}\left(x, t_{r}\right) \prod_{\substack{k=1 \\
k \neq j}}^{m-1}\left(\mu_{j}\left(x, t_{r}\right)-\mu_{k}\left(x, t_{r}\right)\right) .
\end{aligned}
$$

Comparing (3.34) and (3.35), we can obtain (3.29). From (3.19), one can know

$$
\begin{aligned}
& E_{m-1, t_{r}}\left(\mu_{j}\left(x, t_{r}\right), x, t_{r}\right) \\
& \quad=E_{m-1, x}\left(\mu_{j}\left(x, t_{r}\right), x, t_{r}\right) \frac{V_{13}^{(n)} \widetilde{V}_{12}^{(r)}-\widetilde{V}_{13}^{(r)} V_{12}^{(n)}}{V_{13}^{(n)}} \\
& \quad=-\mu_{j, x}\left(x, t_{r}\right) \prod_{k=1}^{m-1}\left(\mu_{j}\left(x, t_{r}\right)-\mu_{k}\left(x, t_{r}\right)\right) \frac{V_{13}^{(n)} \widetilde{V}_{12}^{(r)}-\widetilde{V}_{13}^{(r)} V_{12}^{(n)}}{V_{13}^{(n)}} \\
& =-\varepsilon(m)\left[3 y^{2}\left(\hat{\mu}_{j}\left(x, t_{r}\right)\right)+S_{m}\left(\mu_{j}\left(x, t_{r}\right)\right)\right]\left(V_{13}^{(n)} \widetilde{V}_{12}^{(r)}-\widetilde{V}_{13}^{(r)} V_{12}^{(n)}\right),
\end{aligned}
$$

then we have (3.30). Similarly, we can prove (3.31) and (3.32).

\section{Algebro-geometric solutions to the second-order Benjamin-Ono hierarchy}

In our final and principal section, we obtain Riemann theta function representations for the Baker-Akhiezer function and the meromorphic function; especially, the theta func- 
tion representations for general algebro-geometric solutions $u, v$ of the second-order Benjamin-Ono hierarchy. For the convenience, we assume that the curve $\mathcal{K}_{m-1}$ is nonsingular.

For investigating the asymptotic expansion of $\phi_{1}\left(P, x, t_{r}\right)$ near $P_{\infty}$, we choose the local coordinate $\zeta=\lambda^{-\frac{1}{3}}$, then we get the following lemma.

Lemma 4.1 Let $\left(x, t_{r}\right) \in \mathbb{C}^{2}$, near $P_{\infty} \in \mathcal{K}_{m-1}$, we have

$$
\phi_{1}\left(P, x, t_{r}\right) \underset{\zeta \rightarrow 0}{=} \frac{1}{\zeta} \sum_{j=0}^{\infty} \kappa_{j}\left(x, t_{r}\right) \zeta^{j} \quad \text { as } P \rightarrow P_{\infty}
$$

where

$$
\begin{aligned}
& \kappa_{0}=1, \quad \kappa_{1}=0, \quad \kappa_{2}=\frac{2}{3} u, \quad \kappa_{3}=\frac{1}{3}\left(\nu-u_{x}\right), \\
& \kappa_{4}=\frac{1}{9} u_{x x}-\frac{1}{3} v_{x}, \quad \kappa_{5}=\frac{2}{9}\left(v_{x x}-u u_{x}-u v\right), \\
& \kappa_{j}=-\frac{1}{3}\left[\kappa_{j-2, x x}+3 \sum_{i=2}^{j-1} \kappa_{j-1-i} \kappa_{i, x}+\sum_{i=2}^{j-1} \kappa_{i} \kappa_{j-i}+\sum_{i=2}^{j-1} \sum_{l=0}^{j-i} \kappa_{i} \kappa_{l} \kappa_{j-i-l}-2 u \kappa_{j-2}\right] \quad(j \geq 4) .
\end{aligned}
$$

Proof In terms of the local coordinate $\zeta=\lambda^{-\frac{1}{3}},(3.13)$ reads

$$
\phi_{1, x x}+3 \phi_{1} \phi_{1, x}+\phi_{1}^{3}-2 u \phi_{1}=u_{x}+v+\zeta^{-3} .
$$

Then, by inserting the power series ansatz of $\phi_{1}\left(P, x, t_{r}\right)$ in $\zeta$ as follows:

$$
\phi_{1}\left(P, x, t_{r}\right) \underset{\zeta \rightarrow 0}{=} \frac{1}{\zeta} \sum_{j=0}^{\infty} \kappa_{j}\left(x, t_{r}\right) \zeta^{j}
$$

into (4.3)

$$
\begin{aligned}
& \zeta^{-1} \sum_{j=0}^{\infty} \kappa_{j, x x} \zeta^{j}+3 \zeta^{-2} \sum_{j=0}^{\infty} \sum_{i=0}^{\infty} \kappa_{j} \kappa_{i, x} \zeta^{(j+i)}+\zeta^{-3} \sum_{j=0}^{\infty} \sum_{i=0}^{\infty} \sum_{l=0}^{\infty} \kappa_{j} \kappa_{i} \kappa_{l} \zeta^{(j+i+l)}-2 u \zeta^{-1} \sum_{j=0}^{\infty} \kappa_{j} \zeta^{j} \\
& \quad=u_{x}+v+\zeta^{-3}
\end{aligned}
$$

and comparing the same powers of $\zeta$ in (4.5), we arrive at (4.2).

One infers, from (3.7), (3.25), (3.26), and (4.1), that the divisor $\left(\phi_{1}\left(P, x, t_{r}\right)\right)$ of $\phi_{1}\left(P, x, t_{r}\right)$ is given by

$$
\left(\phi_{1}\left(P, x, t_{r}\right)\right)=\mathcal{D}_{\hat{v}_{0}\left(x, t_{r}\right), \ldots, \hat{v}_{m-1}\left(x, t_{r}\right)}(P)-\mathcal{D}_{P_{\infty}, \hat{\mu}_{1}\left(x, t_{r}\right) \ldots, \hat{\mu}_{m-1}\left(x, t_{r}\right)}(P) .
$$

That is, $\hat{v}_{0}\left(x, t_{r}\right), \ldots, \hat{v}_{m-1}\left(x, t_{r}\right)$ are the $m$ zeros of $\phi_{1}\left(P, x, t_{r}\right)$ and $P_{\infty}, \hat{\mu}_{1}\left(x, t_{r}\right), \ldots, \hat{\mu}_{m-1}\left(x, t_{r}\right)$ are its $m$ poles. 
A straightforward calculation reveals that the asymptotic behaviors of $y(P)$ and $S_{m}(\lambda)$ near $P_{\infty}$ are

$$
\begin{aligned}
& y(P) \underset{\zeta \rightarrow 0}{=} \begin{cases}\zeta^{-3 n-2}\left[1+\alpha_{0} \zeta+\beta_{1} \zeta^{3}+\alpha_{1} \zeta^{4}+O\left(\zeta^{6}\right)\right] & \text { as } P \rightarrow P_{\infty}, m=3 n+2, \\
\zeta^{-3 n-1}\left[1+\beta_{1} \zeta^{2}+\alpha_{1} \zeta^{3}+O\left(\zeta^{5}\right)\right] & \text { as } P \rightarrow P_{\infty}, m=3 n+1,\end{cases} \\
& S_{m}(\lambda) \underset{\zeta \rightarrow 0}{=} \begin{cases}-3 \zeta^{-6 n-3}\left[\alpha_{0}+\left(\alpha_{1}+\beta_{1} \alpha_{0}\right) \zeta^{3}+O\left(\zeta^{6}\right)\right] & \text { as } P \rightarrow P_{\infty}, m=3 n+2, \\
-3 \zeta^{-6 n}\left[\beta_{1}+O\left(\zeta^{3}\right)\right] & \text { as } P \rightarrow P_{\infty}, m=3 n+1 .\end{cases}
\end{aligned}
$$

Next we will introduce the three kinds of holomorphic differentials and show some properties of them. The holomorphic differentials $\eta_{l}(P)$ on $\mathcal{K}_{m-1}$ are defined by

$$
\eta_{l}(P)=\frac{1}{3 y(P)^{2}+S_{m}} \begin{cases}\lambda^{l-1} d \lambda, & 1 \leq l \leq m-n-1 \\ y(P) \lambda^{l+n-m} d \lambda, & m-n \leq l \leq m-1 .\end{cases}
$$

To construct the theta function and normalize the holomorphic differentials, we choose a homology basis $\left\{\mathfrak{a}_{j}, \mathbb{b}_{j}\right\}_{j=1}^{m-1}$ on $\mathcal{K}_{m-1}$ so that they satisfy

$$
\mathbb{a}_{j} \circ \mathbb{b}_{k}=\delta_{j, k}, \quad \mathbb{a}_{j} \circ \mathbb{\mathfrak { d }}_{k}=0, \quad \mathbb{b}_{j} \circ \mathbb{b}_{k}=0, \quad j, k=1, \ldots, m-1 .
$$

Introducing an invertible matrix $E=\left(E_{j, k}\right)_{(m-1) \times(m-1)}$ and $\underline{e}(k)=\left(e_{1}(k), \ldots, e_{m-1}(k)\right)$, where

$$
E_{j, k}=\int_{\rrbracket_{k}} \eta_{j}, \quad e_{j}(k)=\left(E^{-1}\right)_{j, k},
$$

and the normalized holomorphic differentials $\omega_{j}$ for $j=1, \ldots, m-1$,

$$
\begin{aligned}
& \omega_{j}=\sum_{l=1}^{m-1} e_{j}(l) \eta_{l}, \quad \int_{\mathbb{a}_{k}} \omega_{j}=\delta_{j, k}, \\
& \int_{\mathbb{b}_{k}} \omega_{j}=\tau_{j, k} \quad\left(\tau_{j, k}=\tau_{k, j}\right), j, k=1, \ldots, m-1 .
\end{aligned}
$$

Let $\omega_{P_{\infty}, 2}^{(2)}(P)$ denote the normalized second Abel differential defined by

$$
\omega_{P_{\infty}, 2}^{(2)}(P)=-\sum_{j=1}^{m-1} z_{j} \eta_{j}(P)-\frac{1}{3 y(P)^{2}+S_{m}} \begin{cases}\lambda^{2 n} d \lambda, & m=3 n+1 \\ y(P) \lambda^{n} d \lambda, & m=3 n+2\end{cases}
$$

which is holomorphic on $\mathcal{K}_{m-1} \backslash\left\{P_{\infty}\right\}$ with a pole of order 2 at $P_{\infty}$, and the constants $\left\{z_{j}\right\}_{j=1, \ldots, m-1}$ are determined by the normalization condition

$$
\int_{\gtrsim_{j}} \omega_{P_{\infty}, 2}^{(2)}(P)=0, \quad j=1, \ldots, m-1 .
$$

The $\mathbb{b}$-periods of the differential $\omega_{P_{\infty}, 2}^{(2)}$ are denoted by

$$
\underline{U}_{2}^{(2)}=\left(U_{2,1}^{(2)}, \ldots, U_{2, m-1}^{(2)}\right), \quad U_{2, j}^{(2)}=\frac{1}{2 \pi i} \int_{\mathbb{b}_{j}} \omega_{P_{\infty}, 2}^{(2)}(P), \quad j=1, \ldots, m-1
$$


On the other hand, $\omega_{P_{\infty}, 3}^{(2)}(P)$ denotes the normalized third Abel differential which is holomorphic on $\mathcal{K}_{m-1} \backslash\left\{P_{\infty}\right\}$ with a pole of order 3 at $P_{\infty}$

$$
\omega_{P_{\infty}, 3}^{(2)}(P) \underset{\zeta \rightarrow 0}{=}\left(\zeta^{-3}+O(1)\right) d \zeta \quad \text { as } P \rightarrow P_{\infty}
$$

and the $\mathbb{b}$-periods of it are defined by

$$
\underline{U}_{3}^{(2)}=\left(U_{3,1}^{(2)}, \ldots, U_{3, m-1}^{(2)}\right), \quad U_{3, j}^{(2)}=\frac{1}{2 \pi i} \int_{\mathbb{b}_{j}} \omega_{P_{\infty}, 3}^{(2)}, \quad j=1, \ldots, m-1 .
$$

Furthermore, the normalized third Abel differential $\omega_{P_{\infty}, \hat{v}_{0}(x)}^{(3)}(P)$ is holomorphic on $\mathcal{K}_{m-1} \backslash$ $\left\{P_{\infty}, \hat{v}_{0}(x)\right\}$ with simple poles at $P_{\infty}$ and $\hat{v}_{0}(x)$ with residues \pm 1 , respectively, that is,

$$
\begin{aligned}
& \omega_{P_{\infty}, \hat{v}_{0}(x)}^{(3)}(P) \underset{\zeta \rightarrow 0}{=}\left(\zeta^{-1}+O(1)\right) d \zeta \quad \text { as } P \rightarrow P_{\infty}, \\
& \omega_{P_{\infty}, \hat{v}_{0}(x)}^{(3)}(P) \underset{\zeta \rightarrow 0}{=}\left(-\zeta^{-1}+O(1)\right) d \zeta \quad \text { as } P \rightarrow \hat{v}_{0}(x) .
\end{aligned}
$$

Then

$$
\begin{aligned}
& \int_{P_{0}}^{P} \omega_{P_{\infty}, \hat{v}_{0}(x)}^{(3)}(P)=\ln \zeta+e^{(3)}\left(P_{0}\right)+O(\zeta) \quad \text { as } P \rightarrow P_{\infty}, \\
& \int_{P_{0}}^{P} \omega_{P_{\infty}, \hat{\nu}_{0}(x)}^{(3)}(P)=-\ln \zeta+e^{(3)}\left(P_{0}\right)+O(\zeta) \quad \text { as } P \rightarrow \hat{v}_{0}(x)
\end{aligned}
$$

with $e^{(3)}\left(P_{0}\right)$ being an integration constant.

A straightforward Laurent expansion of (4.9), (4.10), and (4.11) near $P_{\infty}$ yields the following results.

Lemma 4.2 Near $P_{\infty}$ in the local coordinate $\zeta=\lambda^{-\frac{1}{3}}$, the differentials $\underline{\omega}$ and $\omega_{P_{\infty}, 2}^{(2)}$ have the Laurent series

$$
\underline{\omega}=\left(\omega_{1}, \ldots, \omega_{m-1}\right) \underset{\zeta \rightarrow 0}{=}\left(\underline{\rho}_{0}+\underline{\rho}_{1} \zeta+\underline{\rho}_{2} \zeta^{3}+O\left(\zeta^{4}\right)\right) d \zeta,
$$

with

$$
\begin{aligned}
& \underline{\rho}_{0}= \begin{cases}-\underline{e}(m-n-1), & m=3 n+2, \\
-\underline{e}(m-1), & m=3 n+1,\end{cases} \\
& \underline{\rho}_{1}= \begin{cases}-\underline{e}(m-1)+\alpha_{0} \underline{e}(m-n-1), & m=3 n+2, \\
-\underline{e}(m-n-1), & m=3 n+1,\end{cases} \\
& \underline{\rho}_{2}= \begin{cases}\left(2 \beta_{1}-\alpha_{0}^{3}\right) \underline{e}(m-n-1)+\alpha_{0}^{2} \underline{e}(m-1)-\underline{e}(m-n-2), & m=3 n+2, \\
\alpha_{1} \underline{e}(m-1)+\beta_{1} \underline{e}(m-n-1)-\underline{e}(m-2), & m=3 n+1,\end{cases} \\
& \omega_{P_{\infty}, 2}^{(2)}(P) \underset{\zeta \rightarrow 0}{=}\left\{\begin{array}{l}
\left(\zeta^{-2}+z_{m-n-1}-\alpha_{0}^{2}+\left(-\beta_{1}+\alpha_{0}^{3}-\alpha_{0} z_{m-n-1}+z_{m-1}\right) \zeta+O\left(\zeta^{2}\right)\right) d \zeta, \\
m=3 n+2, \\
\left(\zeta^{-2}+z_{m-1}-\beta_{1}+\left(z_{m-n-1}-2 \alpha_{1}\right) \zeta+O\left(\zeta^{2}\right)\right) d \zeta, \\
m=3 n+1 .
\end{array}\right.
\end{aligned}
$$


From Lemma 4.2 we infer

$$
\int_{P_{0}}^{P} \omega_{P_{\infty}, 2}^{(2)}(P) \underset{\zeta \rightarrow 0}{=}-\zeta^{-1}+e_{2}^{(2)}\left(P_{0}\right)-q_{1} \zeta+q_{2} \zeta^{2}+O\left(\zeta^{3}\right) \quad \text { as } P \rightarrow P_{\infty}
$$

where $e_{2}^{(2)}\left(P_{0}\right)$ is an appropriate constant, and

$$
\begin{aligned}
& q_{1}= \begin{cases}-z_{m-n-1}+\alpha_{0}^{2}, & m=3 n+2, \\
-z_{m-1}+\beta_{1}, & m=3 n+1,\end{cases} \\
& q_{2}= \begin{cases}\frac{1}{2}\left(-\beta_{1}+\alpha_{0}^{3}-\alpha_{0} z_{m-n-1}+z_{m-1}\right), & m=3 n+2, \\
\frac{1}{2} z_{m-n-1}-\alpha_{1}, & m=3 n+1 .\end{cases}
\end{aligned}
$$

Let $\theta(\underline{\lambda})$ denote the Riemann theta function [20-22] associated with $\mathcal{K}_{m-1}$ and the appropriately fixed homology basis $\left\{\mathbb{a}_{j}, \mathfrak{b}_{j}\right\}_{j=1}^{m-1}$. Next we choose a convenient base point $P_{0} \in \mathcal{K}_{m-1} \backslash\left\{P_{\infty}\right\}$. For brevity, define the function $\underline{\lambda}: \mathcal{K}_{m-1} \times \sigma^{m-1} \mathcal{K}_{m-1} \rightarrow \mathbb{C}$ by

$$
\begin{aligned}
& \underline{\lambda}(P, \underline{Q})=\underline{\Xi}_{P_{0}}-\underline{A}_{P_{0}}(P)+\underline{\alpha}_{P_{0}}\left(\mathcal{D}_{\underline{Q}}\right), \quad P \in \mathcal{K}_{m-1}, \\
& \underline{Q}=\left(Q_{1}, \ldots, Q_{m-1}\right) \in \sigma^{m-1} \mathcal{K}_{m-1},
\end{aligned}
$$

where $\underline{\Xi}_{P_{0}}$ is the vector of Riemann constants, and the Abel maps $\underline{A}_{P_{0}}(P)$ and $\underline{\alpha}_{P_{0}}(P)$ are defined by (period lattice $L_{m-1}=\left\{\underline{z} \in \mathbb{C}^{m-1} \mid \underline{z}=\underline{N}+\tau \underline{M}, \underline{N}, \underline{M} \in \mathbb{Z}^{m-1}\right\}$ )

$$
\begin{aligned}
& \underline{A}_{P_{0}}: \mathcal{K}_{m-1} \rightarrow \mathcal{J}\left(\mathcal{K}_{m-1}\right)=\mathbb{C}^{m-1} / L_{m-1}, \\
& P \mapsto \underline{A}_{P_{0}}(P)=\left(A_{P_{0}, 1}(P), \ldots, A_{P_{0}, m-1}(P)\right)=\left(\int_{P_{0}}^{P} \omega_{1}, \ldots, \int_{P_{0}}^{P} \omega_{m-1}\right)\left(\bmod L_{m-1}\right),
\end{aligned}
$$

and

$$
\begin{aligned}
& \underline{\alpha}_{P_{0}}: \operatorname{Div}\left(\mathcal{K}_{m-1}\right) \rightarrow \mathcal{J}\left(\mathcal{K}_{m-1}\right), \\
& \mathcal{D} \mapsto \underline{\alpha}_{P_{0}}(\mathcal{D})=\sum_{P \in \mathcal{K}_{m-1}} \mathcal{D}(P) \underline{A}_{P_{0}}(P) .
\end{aligned}
$$

In view of these preparations, we give the theta function representation of our fundamental object $\phi_{1}\left(P, x, t_{r}\right)$.

Theorem 4.3 Let $P=(\lambda, y) \in \mathcal{K}_{m-1} \backslash\left\{P_{\infty}\right\}$, and let $\left(x, t_{r}\right),\left(x_{0}, t_{0, r}\right) \in \Omega_{\mu}$, where $\Omega_{\mu} \subseteq \mathbb{C}^{2}$ is open and connected. Suppose also that $\mathcal{D}_{\underline{\hat{\mu}}\left(x, t_{r}\right)}$, or equivalently, $\mathcal{D}_{\underline{\hat{v}}\left(x, t_{r}\right)}$ is nonspecial for $\left(x, t_{r}\right) \in \Omega_{\mu}$. Then

$$
\phi_{1}\left(P, x, t_{r}\right)=\frac{\theta\left(\underline{\lambda}\left(P, \underline{\hat{v}}\left(x, t_{r}\right)\right)\right) \theta\left(\underline{\lambda}\left(P_{\infty}, \underline{\hat{\mu}}\left(x, t_{r}\right)\right)\right)}{\theta\left(\underline{\lambda}\left(P_{\infty}, \underline{\hat{v}}\left(x, t_{r}\right)\right)\right) \theta\left(\underline{\lambda}\left(P, \underline{\hat{\mu}}\left(x, t_{r}\right)\right)\right)} \exp \left(e^{(3)}\left(P_{0}\right)-\int_{P_{0}}^{P} \omega_{P_{\infty}, \hat{v}_{0}\left(x, t_{r}\right)}^{(3)}\right) .
$$

Proof Let $\Phi$ denote the right-hand side of (4.20). From (4.15) it follows that

$$
\exp \left(e^{(3)}\left(P_{0}\right)-\int_{P_{0}}^{P} \omega_{P_{\infty}, \hat{v}_{0}\left(x, t_{r}\right)}^{(3)}\right) \underset{\zeta \rightarrow 0}{=} \zeta^{-1}+O(1)
$$


Using (4.6) we immediately know that $\phi_{1}$ has simple poles at $\underline{\hat{\mu}}\left(x, t_{r}\right)$ and $P_{\infty}$, and simple zeros at $\hat{v}_{0}\left(x, t_{r}\right), \underline{\hat{v}}\left(x, t_{r}\right)$. By $(4.20)$ and the Riemann vanishing theorem, we see that $\Phi$ has the same properties. Using the Riemann-Roch theorem [21, 22], we conclude that the holomorphic function $\frac{\Phi}{\phi_{1}}=\gamma$, where $\gamma$ is a constant. Using (4.21) and Lemma 4.1, we have

$$
\frac{\Phi}{\phi_{1}} \underset{\zeta \rightarrow 0}{=} \frac{(1+O(\zeta))\left(\zeta^{-1}+O(1)\right)}{\zeta^{-1}+O(\zeta)} \underset{\zeta \rightarrow 0}{=} 1+O(\zeta) \text { as } P \rightarrow P_{\infty}
$$

from which we conclude $\gamma=1$.

Let $\omega_{P_{\infty}, s}^{(2)}, s=3 r+2$ (or $\left.3 r+1\right), r \in \mathbb{N}_{0}$, be the normalized differential of the second kind holomorphic on $\mathcal{K}_{m-1} \backslash\left\{P_{\infty}\right\}$, with a pole of order $s$ at $P_{\infty}$,

$$
\omega_{P_{\infty}, S}^{(2)}(P) \underset{\zeta \rightarrow 0}{=}\left(\zeta^{-s}+O(1)\right) d \zeta \quad \text { as } P \rightarrow P_{\infty}
$$

Then we define the normalized differentials as

$$
\begin{aligned}
\widetilde{\Omega}_{P_{\infty}, s+1}^{(2)} & =\sum_{l=0}^{r} \tilde{\beta}_{r-l}(3 l+2) \widetilde{\omega}_{P_{\infty}, 3 l+3}^{(2)}+\sum_{l=0}^{r} \tilde{\alpha}_{r-l}(3 l+1) \widetilde{\omega}_{P_{\infty}, 3 l+2}^{(2)}, \\
s=3 r & +2(\text { or } 3 r+1), r \in \mathbb{N}_{0},
\end{aligned}
$$

where

$$
\left(\tilde{\alpha}_{0}, \tilde{\beta}_{0}\right)=\left\{\begin{array}{ll}
\left(\tilde{\alpha}_{0}, 1\right), & s=3 r+2, \\
(1,0), & s=3 r+1,
\end{array} \quad \tilde{\alpha}_{0} \in \mathbb{C} .\right.
$$

In addition, we define the vector of $\mathbb{b}$-periods of them as

$$
\begin{gathered}
\widetilde{U}_{s+1}^{(2)}=\left(\widetilde{U}_{s+1,1}^{(2)}, \ldots, \widetilde{U}_{s+1, m-1}^{(2)}\right), \quad \widetilde{U}_{s+1, j}^{(2)}=\frac{1}{2 \pi i} \int_{\mathbb{b}_{j}} \widetilde{\Omega}_{P_{\infty}, s+1}^{(2)}, \\
j=1, \ldots, m-1, s=3 r+2(\text { or } 3 r+1), r \in \mathbb{N}_{0} .
\end{gathered}
$$

Motivated by the second integration in (3.23), one defines the function $I_{s}\left(P, x, t_{r}\right)$, meromorphic on $\mathcal{K}_{m-1} \times \mathbb{C}^{2}$, by

$$
\begin{aligned}
I_{s}\left(P, x, t_{r}\right)= & \widetilde{V}_{11}^{(r)}\left(\lambda, x, t_{r}\right)+\widetilde{V}_{12}^{(r)}\left(\lambda, x, t_{r}\right) \phi_{1}\left(P, x, t_{r}\right)+\widetilde{V}_{13}^{(r)}\left(\lambda, x, t_{r}\right)\left(\phi_{1, x}\left(P, x, t_{r}\right)\right. \\
& \left.+\phi_{1}^{2}\left(P, x, t_{r}\right)-u\left(x, t_{r}\right)\right) .
\end{aligned}
$$

Denote by $\bar{I}_{s}\left(P, x, t_{r}\right)$ the associated homogeneous one replacing $\widetilde{V}_{1 j}^{(r)}$ by $\overline{\widetilde{V}}_{1 j}^{(r)}$, where

$$
\tilde{\widetilde{V}}_{1 j}^{(r)}=\left\{\begin{array}{ll}
\left.\widetilde{V}_{1 j}^{(r)}\right|_{\tilde{\alpha}_{0}=1, \tilde{\alpha}_{1}=\cdots=\tilde{\alpha}_{r}=\tilde{\beta}_{0}=\tilde{\beta}_{1}=\cdots=\tilde{\beta}_{r}=0}, \quad s=3 r+1, \\
\left.\widetilde{V}_{1 j}^{(r)}\right|_{\tilde{\beta}_{0}=1, \tilde{\alpha}_{0}=\tilde{\alpha}_{1}=\cdots=\tilde{\alpha}_{r}=\tilde{\beta}_{1}=\cdots=\tilde{\beta}_{r}=0}, \quad s=3 r+2,
\end{array} \quad j=1,2,3 .\right.
$$

Lemma 4.4 Let $s=3 r+2($ or $3 r+1), r \in \mathbb{N}_{0},\left(x, t_{r}\right) \in \mathbb{C}^{2}$, and $\lambda=\zeta^{-3}$ be the local coordinate near $P_{\infty}$. Then

$$
\bar{I}_{s}\left(P, x, t_{r}\right) \underset{\zeta \rightarrow 0}{=}-\zeta^{-s}+O(\zeta) \quad \text { as } P \rightarrow P_{\infty}
$$


Proof For the sake of convenience, we introduce the notation $\widetilde{V}_{1 j}^{(r, s)}=\widetilde{V}_{1 j}^{(r)}, j=1,2,3$. From (2.12) and (4.25), one easily gets

$$
\begin{aligned}
\bar{I}_{s}\left(P, x, t_{r}\right)= & \overline{\widetilde{V}}_{11}^{(r, s)}\left(\lambda, x, t_{r}\right)+\overline{\widetilde{V}}_{12}^{(r, s)}\left(\lambda, x, t_{r}\right) \phi_{1}\left(P, x, t_{r}\right) \\
& +\overline{\widetilde{V}}_{13}^{(r, s)}\left(\lambda, x, t_{r}\right)\left(\phi_{1, x}\left(P, x, t_{r}\right)+\phi_{1}^{2}\left(P, x, t_{r}\right)-u\right) \\
= & \frac{1}{6} \overline{\tilde{b}}_{x x}^{(r, s)}\left(\lambda, x, t_{r}\right)-\frac{1}{3} u \overline{\tilde{b}}^{(r, s)}\left(\lambda, x, t_{r}\right)-\overline{\tilde{a}}_{x}^{(r, s)}\left(\lambda, x, t_{r}\right) \\
& -\left[\overline{\tilde{\tilde{a}}}^{(r, s)}\left(\lambda, x, t_{r}\right)-\frac{1}{2} \overline{\tilde{b}}_{x}^{(r, s)}\left(\lambda, x, t_{r}\right)\right] \phi_{1}\left(P, x, t_{r}\right) \\
& +\overline{\tilde{b}}^{(r, s)}\left[\phi_{1, x}\left(P, x, t_{r}\right)+\phi_{1}^{2}\left(P, x, t_{r}\right)-u\left(x, t_{r}\right)\right] .
\end{aligned}
$$

From (4.1), we can see

$$
\begin{aligned}
& \bar{I}_{1}=\phi_{3}\left(P, x, t_{r}\right)=\zeta^{-1}+O(\zeta), \\
& \bar{I}_{2}=-\frac{1}{3} u\left(x, t_{r}\right)+\phi_{1, x}\left(P, x, t_{r}\right)-\phi_{1}^{2}\left(P, x, t_{r}\right)-u\left(x, t_{r}\right)=\zeta^{-2}+O(\zeta) .
\end{aligned}
$$

So (4.26) is correct for $s=1$ and $s=2$. Then one may rewrite (4.26) as

$$
\bar{I}_{s}\left(P, x, t_{r}\right) \underset{\zeta \rightarrow 0}{=} \zeta^{-s}+\sum_{j=1}^{\infty} \delta_{j}\left(x, t_{r}\right) \zeta^{j} \quad \text { as } P \rightarrow P_{\infty}
$$

for some coefficients $\left\{\delta_{j}\left(x, t_{r}\right)\right\}_{j \in \mathbb{N}}$. From (3.20) and (4.25), we can see

$$
\begin{aligned}
\partial_{x} \bar{I}_{s}\left(P, x, t_{r}\right) \\
=\partial_{x}\left(\overline{\widetilde{V}}_{12}^{(r, s)}\left(\lambda, x, t_{r}\right) \phi_{1}\left(P, x, t_{r}\right)+\overline{\widetilde{V}}_{13}^{(r, s)}\left(\lambda, x, t_{r}\right)\left(\phi_{1, x}\left(P, x, t_{r}\right)+\phi_{1}^{2}\left(P, x, t_{r}\right)-u\right)\right. \\
\left.\quad+\overline{\widetilde{V}}_{11}^{(r, s)}\left(\lambda, x, t_{r}\right)\right) \\
=\phi_{1, t_{r}}\left(P, x, t_{r}\right),
\end{aligned}
$$

that is,

$$
\partial_{x}\left(-\zeta^{-s}+\sum_{j=1}^{\infty} \delta_{j}\left(x, t_{r}\right) \zeta^{j}\right)=\left(\zeta^{-1}+\sum_{j=1}^{\infty} \kappa_{j}\left(x, t_{r}\right) \zeta^{j-1}\right)_{t_{r}}=\left(\sum_{j=1}^{\infty} \kappa_{j+1}\left(x, t_{r}\right) \zeta^{j}\right)_{t_{r}} .
$$

Using (3.2), (4.2), and comparing coefficients of $\zeta$ in (4.28), we should obtain

$$
\begin{aligned}
& \delta_{j, x}\left(x, t_{r}\right)=\kappa_{j+1, t_{r}}\left(x, t_{r}\right), \quad j=1,2, \ldots \\
& \delta_{1, x}\left(x, t_{r}\right)=\kappa_{2, t_{r}}\left(x, t_{r}\right)=\frac{2}{3} u_{t_{r}}\left(x, t_{r}\right)=-\overline{\tilde{b}}_{r, x}^{(r, s)}\left(x, t_{r}\right), \\
& \delta_{2, x}\left(x, t_{r}\right)=\kappa_{3, t_{r}}\left(x, t_{r}\right)=\frac{1}{3}\left(-u\left(x, t_{r}\right)+v\left(x, t_{r}\right)\right)_{t_{r}}=\frac{1}{2} \overline{\tilde{b}}_{r, x x}^{(r, s)}\left(x, t_{r}\right)-\overline{\tilde{a}}_{r, x}^{(r, s)}\left(x, t_{r}\right), \\
& \delta_{3, x}\left(x, t_{r}\right)=\kappa_{4, t_{r}}\left(x, t_{r}\right)=\left(\frac{1}{9} u_{x x}\left(x, t_{r}\right)-\frac{1}{3} v_{x}\left(x, t_{r}\right)\right)_{t_{r}}=-\frac{1}{6} \overline{\tilde{b}}_{r, x x x}^{(r, s)}\left(x, t_{r}\right)+\overline{\tilde{a}}_{r, x x}^{(r, s)}\left(x, t_{r}\right) .
\end{aligned}
$$


That is,

$$
\begin{aligned}
& \delta_{1}\left(x, t_{r}\right)=\gamma_{1}\left(t_{r}\right)-\overline{\tilde{b}}_{r}^{(r, s)}\left(x, t_{r}\right), \\
& \delta_{2}\left(x, t_{r}\right)=\gamma_{2}\left(t_{r}\right)+\frac{1}{2} \overline{\tilde{b}}_{r, x}^{(r, s)}\left(x, t_{r}\right)-\overline{\tilde{a}}_{r}^{(r, s)}\left(x, t_{r}\right), \\
& \delta_{3}\left(x, t_{r}\right)=\gamma_{3}\left(t_{r}\right)-\frac{1}{6} \overline{\tilde{b}}_{r, x x}^{(r, s)}\left(x, t_{r}\right)+\overline{\tilde{a}}_{r, x}^{(r, s)}\left(x, t_{r}\right),
\end{aligned}
$$

with $\gamma_{1}\left(t_{r}\right), \gamma_{2}\left(t_{r}\right), \gamma_{3}\left(t_{r}\right)$ being integration constants. From the definition of $\bar{I}_{s}$, the power series for $\phi_{1}\left(P, x, t_{r}\right)$ and the coefficients of $\overline{\tilde{a}}\left(\zeta, x, t_{r}\right), \overline{\tilde{b}}\left(\zeta, x, t_{r}\right)$, we deduce that $\gamma_{1}\left(t_{r}\right)=$ $\gamma_{2}\left(t_{r}\right)=\gamma_{3}\left(t_{r}\right)=0$. Hence one concludes

$$
\begin{aligned}
\bar{I}_{s}\left(P, x, t_{r}\right)= & \zeta^{-s}-\overline{\tilde{b}}_{r}^{(r, s)} \zeta+\left(\frac{1}{2} \overline{\tilde{b}}_{r, x}^{(r, s)}-\overline{\tilde{a}}_{r}^{(r, s)}\right) \zeta^{2}+\left(-\frac{1}{6} \overline{\tilde{b}}_{r, x x}^{(r, s)}+\overline{\tilde{\tilde{a}}}_{r, x}^{(r, s)}\right) \zeta^{3} \\
& +O\left(\zeta^{4}\right) \quad \text { as } P \rightarrow P_{\infty} .
\end{aligned}
$$

On the other hand, we will get

$$
\begin{aligned}
\bar{I}_{s+3}\left(P, x, t_{r}\right)= & \zeta^{-3} \bar{I}_{s}+\left(\overline{\tilde{a}}_{r}^{(r+1, s+3)}-\frac{1}{2} \overline{\tilde{b}}_{r, x}^{(r+1, s+3)}\right) \phi_{1}+\overline{\tilde{b}}_{r}^{(r+1, s+3)}\left(\phi_{1, x}+\phi_{1}^{2}-u\right) \\
& +\frac{1}{6} \overline{\tilde{b}}_{r, x x}^{(r+1, s+3)}-\frac{1}{3} u \overline{\tilde{b}}_{r}^{(r+1, s+3)}-\overline{\tilde{a}}_{r, x}^{(r+1, s+3)} \\
= & \zeta^{-s-3}+O(\zeta) .
\end{aligned}
$$

By (3.1) one knows that

$$
\begin{aligned}
I_{s}\left(P, x, t_{r}\right)= & \sum_{l=0}^{r} \tilde{\beta}_{r-l} \bar{I}_{3 l+2}\left(P, x, t_{r}\right) \\
& +\sum_{l=0}^{r} \tilde{\alpha}_{r-l} \bar{I}_{3 l+1}\left(P, x, t_{r}\right), \quad s=3 r+2(\text { or } s=3 r+1) .
\end{aligned}
$$

Thus

$$
\begin{gathered}
\int_{t_{0, r}}^{t_{r}} I_{s}(P, x, \tau) d \tau \underset{\zeta \rightarrow 0}{=}\left(t_{r}-t_{0, r}\right) \sum_{l=0}^{r}\left(\tilde{\beta}_{r-l} \frac{1}{\zeta^{3 l+2}}+\tilde{\alpha}_{r-l} \frac{1}{\zeta^{3 l+1}}\right) \\
+O(\zeta) \quad \text { as } P \rightarrow P_{\infty} .
\end{gathered}
$$

Furthermore, integrating (4.23) yields

$$
\begin{aligned}
& \int_{P_{0}}^{P} \widetilde{\Omega}_{P_{\infty}, s+1}^{(2)} \\
& \quad=\sum_{l=0}^{r} \tilde{\beta}_{r-l}(3 l+2) \int_{\zeta_{0}}^{\zeta} \widetilde{\omega}_{P_{\infty}, 3 l+3}^{(2)}+\sum_{l=0}^{r} \tilde{\alpha}_{r-l}(3 l+1) \int_{\zeta_{0}}^{\zeta} \widetilde{\omega}_{P_{\infty}, 3 l+2}^{(2)}
\end{aligned}
$$




$$
\begin{aligned}
& \underset{\zeta \rightarrow 0}{=} \sum_{l=0}^{r} \tilde{\beta}_{r-l}(3 l+2) \int_{\zeta 0}^{\zeta} \frac{1}{\zeta^{3 l+3}} d \zeta+\sum_{l=0}^{r} \tilde{\alpha}_{r-l}(3 l+1) \int_{\zeta_{0}}^{\zeta} \frac{1}{\zeta^{3 l+2}} d \zeta+O(\zeta) \\
& \underset{\zeta \rightarrow 0}{=}-\sum_{l=0}^{r} \tilde{\beta}_{r-l} \frac{1}{\zeta^{3 l+2}}-\sum_{l=0}^{r} \tilde{\alpha}_{r-l} \frac{1}{\zeta^{3 l+1}}+e_{s+1}^{(2)}\left(P_{0}\right)+O(\zeta) \text { as } P \rightarrow P_{\infty}
\end{aligned}
$$

where $e_{s+1}^{(2)}\left(P_{0}\right)$ is a constant. Combing (4.34) and (4.35) indicates

$$
\int_{t_{0, r}}^{t_{r}} I_{s}(P, x, \tau) d \tau \underset{\zeta \rightarrow 0}{=}\left(t_{r}-t_{0, r}\right)\left(e_{s+1}^{(2)}\left(P_{0}\right)-\int_{P_{0}}^{P} \widetilde{\Omega}_{P_{\infty}, s+1}^{(2)}\right)+O(\zeta) \quad \text { as } P \rightarrow P_{\infty} .
$$

Given these preparations, the theta function representation of $\psi_{1}\left(P, x, x_{0}, t_{r}, t_{0, r}\right)$ reads as follows.

Theorem 4.5 Let $P=(\lambda, y) \in \mathcal{K}_{m-1} \backslash\left\{P_{\infty}\right\}$ and let $\left(x, t_{r}\right),\left(x_{0}, t_{0, r}\right) \in \Omega_{\mu}$, where $\Omega_{\mu} \subseteq \mathbb{C}^{2}$ is open and connected. Suppose that $\mathcal{D}_{\underline{\hat{\mu}}\left(x, t_{r}\right)}$, or equivalently, $\mathcal{D}_{\underline{\hat{\hat{v}}}\left(x, t_{r}\right)}$ is nonspecial for $\left(x, t_{r}\right) \in \Omega_{\mu}$. Then

$$
\begin{aligned}
\psi_{1}\left(P, x, x_{0}, t_{r}, t_{0, r}\right)= & \frac{\left.\theta \underline{(} \underline{\lambda}\left(P, \underline{\hat{\mu}}\left(x, t_{r}\right)\right)\right) \theta\left(\underline{\lambda}\left(P_{\infty}, \underline{\hat{\mu}}\left(x_{0}, t_{0, r}\right)\right)\right)}{\theta\left(\underline{\lambda}\left(P_{\infty}, \underline{\hat{\mu}}\left(x, t_{r}\right)\right)\right) \theta\left(\underline{\lambda}\left(P, \underline{\hat{\mu}}\left(x_{0}, t_{0, r}\right)\right)\right)} \\
& \times \exp \left(\left(x-x_{0}\right)\left(e_{2}^{(2)}\left(P_{0}\right)-\int_{P_{0}}^{P} \omega_{P_{\infty}, 2}^{(2)}\right)\right. \\
& \left.+\left(t_{r}-t_{0, r}\right)\left(e_{s+1}^{(2)}\left(P_{0}\right)-\int_{P_{0}}^{P} \tilde{\Omega}_{P_{\infty}, s+1}^{(2)}\right)\right) .
\end{aligned}
$$

Proof Let $\psi_{1}\left(P, x, x_{0}, t_{r}, t_{0, r}\right)$ be defined as in (3.23) and denote the right-hand side of (4.37) by $\Psi\left(P, x, x_{0}, t_{r}, t_{0, r}\right)$. In order to prove that $\psi_{1}=\Psi$, one uses (3.7), (3.12), (3.29), (3.30) and

$$
V_{12}^{(n)} \phi_{1}+V_{13}^{(n)}\left(\phi_{1, x}+\phi_{1}^{2}-u\right)+V_{11}^{(n)}=y
$$

to compute

$$
\begin{aligned}
\phi_{1}\left(P, x, t_{r}\right)= & \frac{y^{2} V_{13}^{(n)}-y A_{m}+B_{m}}{-\varepsilon(m) E_{m-1}} \\
= & \frac{y^{2} V_{13}^{(n)}-y A_{m}+\frac{2}{3} V_{13}^{(n)} S_{m}-\frac{1}{3} \varepsilon(m) E_{m-1, x}}{-\varepsilon(m) E_{m-1}} \\
= & \frac{2}{3} V_{13}^{(n)} \frac{3 y^{2}+S_{m}}{-\varepsilon(m) E_{m-1}}+\frac{1}{3} \partial_{x} \ln E_{m-1}+\frac{V_{13}^{(n)} y\left(y+\frac{A_{m}}{V_{13}^{(n)}}\right)}{\varepsilon(m) E_{m-1}} \\
= & -\frac{\mu_{j, x}}{\lambda-\mu_{j}}+O(1) \\
\lambda \rightarrow \mu_{j}\left(x, t_{r}\right) & =\mu_{j}^{\left(x, t_{r}\right)} \partial_{x} \ln \left(\lambda-\mu_{j}\left(x, t_{r}\right)\right)+O(1), \\
I_{s}\left(P, x, t_{r}\right)= & \widetilde{V}_{12}^{(r)} \phi_{1}+\widetilde{V}_{13}^{(r)}\left(\phi_{1, x}+\phi_{1}^{2}-u\right)+\widetilde{V}_{11}^{(r)} \\
= & \left(\widetilde{V}_{12}^{(r)}-\widetilde{V}_{13}^{(r)} \frac{V_{2}^{(n)}}{V_{13}^{(n)}}\right) \phi_{1}+\widetilde{V}_{11}^{(r)}-\widetilde{V}_{13}^{(r)} \frac{V_{11}^{(n)}}{V_{13}^{(n)}}+y \frac{\widetilde{V}_{13}^{(r)}}{V_{13}^{(n)}} \\
& +\widetilde{V}_{11}^{(r)}-\widetilde{V}_{13}^{(r)} \frac{V_{11}^{(n)}}{V_{13}^{(n)}}+y \frac{\widetilde{V}_{13}^{(r)}}{V_{13}^{(n)}}
\end{aligned}
$$




$$
\begin{aligned}
&=\frac{1}{3} \frac{E_{m-1, t_{r}}}{E_{m-1}}+\left(\widetilde{V}_{12}^{(r)}-\widetilde{V}_{13}^{(r)} \frac{V_{12}^{(n)}}{V_{13}^{(n)}}\right) \frac{y^{2} V_{13}^{(n)}-y A_{m}+\frac{2}{3} S_{m} V_{13}^{(n)}}{-\varepsilon(m) E_{m-1}}+y \frac{\widetilde{V}_{13}^{(r)}}{V_{13}^{(n)}} \\
&=\underset{\lambda \rightarrow \mu_{j}\left(x, t_{r}\right)}{=}-\frac{\mu_{j, t_{r}}}{\lambda-\mu_{j}}+O(1) \\
& \underset{\lambda \rightarrow \mu_{j}\left(x, t_{r}\right)}{=} \partial_{t_{r}} \ln \left(\lambda-\mu_{j}\left(x, t_{r}\right)\right)+O(1) \quad \text { as } P \rightarrow \hat{\mu}_{j}\left(x, t_{r}\right) .
\end{aligned}
$$

Hence

$$
\begin{aligned}
\psi_{1}\left(P, x, x_{0}, t_{r}, t_{0, r}\right) \\
=\frac{\lambda-\mu_{j}\left(x, t_{r}\right)}{\lambda-\mu_{j}\left(x_{0}, t_{r}\right)} \frac{\lambda-\mu_{j}\left(x_{0}, t_{r}\right)}{\lambda-\mu_{j}\left(x_{0}, t_{0, r}\right)} O(1) \\
= \begin{cases}\left(\lambda-\mu_{j}\left(x, t_{r}\right)\right) O(1) & \text { for } P \text { near } \hat{\mu}_{j}\left(x, t_{r}\right) \neq \hat{\mu}_{j}\left(x_{0}, t_{0, r}\right), \\
O(1) & \text { for } P \text { near } \hat{\mu}_{j}\left(x, t_{r}\right)=\hat{\mu}_{j}\left(x_{0}, t_{0, r}\right), \\
\left(\lambda-\mu_{j}\left(x_{0}, t_{0, r}\right)\right)^{-1} O(1) & \text { for } P \text { near } \hat{\mu}_{j}\left(x_{0}, t_{0, r}\right) \neq \hat{\mu}_{j}\left(x, t_{r}\right),\end{cases}
\end{aligned}
$$

where $O(1) \neq 0$ in (4.38). Consequently, all zeros and poles of $\psi_{1}$ and $\Psi$ on $\mathcal{K}_{m-1} \backslash\left\{P_{\infty}\right\}$ are simple and coincide. It remains to identify the essential singularity of $\psi_{1}$ and $\Psi$ at $P_{\infty}$. By (4.35) we see that the singularities in the exponential terms of $\psi_{1}$ and $\Psi$ coincide. The uniqueness result for Baker-Akhiezer functions completes the proof that $\psi_{1}=\Psi$ on $\Omega_{\mu}$.

The straightening out of the second-order Benjiamin-Ono flows by the Abel map is showed in our next result.

Theorem 4.6 Let $\left(x, t_{r}\right),\left(x_{0}, t_{0, r}\right) \in \mathbb{C}^{2}$. Then

$$
\begin{aligned}
& \underline{\alpha}_{P_{0}}\left(\mathcal{D}_{\hat{\hat{\mu}}\left(x, t_{r}\right)}\right)=\underline{\alpha}_{P_{0}}\left(\mathcal{D}_{\hat{\hat{\mu}}\left(x_{0}, t_{0, r}\right)}\right)+\underline{U}_{2}^{(2)}\left(x-x_{0}\right)+\underline{\widetilde{U}}_{s+1}^{(2)}\left(t_{r}-t_{0, r}\right), \\
& \underline{\alpha}_{P_{0}}\left(\mathcal{D}_{\hat{\nu}_{0}\left(x, t_{r}\right) \hat{\hat{\hat{v}}}\left(x, t_{r}\right)}\right)=\underline{\alpha}_{P_{0}}\left(\mathcal{D}_{\hat{v}_{0}\left(x_{0}, t_{0, r}\right) \underline{\hat{\hat{v}}}\left(x_{0}, t_{0, r}\right)}\right)+\underline{U}_{2}^{(2)}\left(x-x_{0}\right)+\underline{\widetilde{U}}_{s+1}^{(2)}\left(t_{r}-t_{0, r}\right) .
\end{aligned}
$$

Our main result, the theta function representation of the algebro-geometric solutions of the second-order Benjamin-Ono hierarchy, now quickly follows.

Theorem 4.7 Let $\left(x, t_{r}\right) \in \Omega_{\mu}$, where $\Omega_{\mu} \subseteq \mathbb{C}^{2}$ is open and connected. Suppose also that $\mathcal{D}_{\underline{\hat{\mu}}\left(x, t_{r}\right)}$, or equivalently, $\mathcal{D}_{\underline{\hat{\hat{v}}}\left(x, t_{r}\right)}$ is nonspecial for $\left(x, t_{r}\right) \in \Omega_{\mu}$. Then

$$
\begin{aligned}
& u\left(x, t_{r}\right)=-\frac{3}{2} \partial_{x}^{2} \ln \left(\theta\left(\underline{\lambda}\left(P_{\infty}, \underline{\hat{\mu}}\left(x, t_{r}\right)\right)\right)\right)+\frac{3}{2} q_{1}, \\
& v\left(x, t_{r}\right)=-3 \partial_{x} \partial_{\underline{u}_{3}^{(2)}} \ln \left(\theta\left(\underline{\lambda}\left(P_{\infty}, \underline{\hat{\mu}}\left(x, t_{r}\right)\right)\right)\right)-3 q_{2},
\end{aligned}
$$

with $q_{1}$ and $q_{2}$ defined in (4.19), and $\partial_{\underline{u}_{3}^{(2)}}$ denotes the directional derivative $\partial_{\underline{u}_{3}^{(2)}}=$ $\sum_{j=1}^{m-1} U_{3, j}^{(2)} \frac{\partial}{\partial \lambda_{j}}$. 
Proof Using Theorem 4.5, one can write $\psi_{1}$ near $P_{\infty}$ in the coordinate $\zeta$ as

$$
\begin{aligned}
& \psi_{1}\left(P, x, x_{0}, t_{r}, t_{0, r}\right) \\
& \underset{\zeta \rightarrow 0}{=}\left(1+\sigma_{1}\left(x, t_{r}\right) \zeta+\sigma_{2}\left(x, t_{r}\right) \zeta^{2}+O\left(\zeta^{3}\right)\right) \exp \left[( x - x _ { 0 } ) \left(\zeta^{-1}+q_{1} \zeta\right.\right. \\
& \left.\left.\quad-q_{2} \zeta^{2}+O\left(\zeta^{3}\right)\right)+\left(t_{r}-t_{0, r}\right) \sum_{l=0}^{r}\left(\tilde{\beta}_{r-l} \frac{1}{\zeta^{3 l+2}}+\tilde{\alpha}_{r-l} \frac{1}{\zeta^{3 l+1}}\right)+O(\zeta)\right],
\end{aligned}
$$

where the terms $\sigma_{1}\left(x, t_{r}\right)$ and $\sigma_{2}\left(x, t_{r}\right)$ in (4.41) come from the Taylor expansion about $P_{\infty}$ of the ratios of the theta functions in (4.37). That is,

$$
\begin{aligned}
& \frac{\theta\left(\underline{\lambda}\left(P, \underline{\hat{\mu}}\left(x, t_{r}\right)\right)\right)}{\theta\left(\underline{\lambda}\left(P_{\infty}, \underline{\hat{\mu}}\left(x, t_{r}\right)\right)\right)} \\
& \underset{\zeta \rightarrow 0}{=} \frac{\theta\left(\underline{\Xi}_{P_{0}}-\underline{A}_{P_{0}}(P)+\underline{\alpha}_{P_{0}}\left(\mathcal{D}_{\underline{\hat{\mu}}\left(x, t_{r}\right)}\right)\right)}{\theta\left(\underline{\Xi}_{P_{0}}-\underline{A}_{P_{0}}\left(P_{\infty}\right)+\underline{\alpha}_{P_{0}}\left(\mathcal{D}_{\hat{\mu}\left(x, t_{r}\right)}\right)\right)} \\
& \underset{\zeta \rightarrow 0}{=} \frac{\theta\left(\ldots, \Xi_{P_{0}, j}-A_{P_{0}, j}\left(P_{\infty}\right)+\alpha_{P_{0}, j}\left(\mathcal{D}_{\hat{\mu}\left(x, t_{r}\right)}\right)-\rho_{0, j} \zeta-\frac{1}{2} \rho_{1, j} \zeta^{2}-\frac{1}{4} \rho_{2, j} \zeta^{4}+O\left(\zeta^{5}\right), \ldots\right)}{\theta\left(\underline{\Xi}_{P_{0}}-\underline{A}_{P_{0}}\left(P_{\infty}\right)+\underline{\alpha}_{P_{0}}\left(\mathcal{D}_{\hat{\mu}\left(x, t_{r}\right)}\right)\right)} \\
& \underset{\zeta \rightarrow 0}{=} \frac{\theta_{0}-\sum_{j=1}^{m-1} \frac{\partial \theta_{0}}{\partial \lambda_{j}} \rho_{0, j} \zeta-\frac{1}{2} \sum_{j=1}^{m-1}\left(\frac{\partial \theta_{0}}{\partial \lambda_{j}} \rho_{1, j}-\sum_{k=1}^{m-1} \frac{\partial^{2} \theta_{0}}{\partial \lambda_{j} \partial \lambda_{k}} \rho_{0, j} \rho_{0, k}\right) \zeta^{2}+O\left(\zeta^{3}\right)}{\theta\left(\underline{\Xi}_{P_{0}}-\underline{A}_{P_{0}}\left(P_{\infty}\right)+\underline{\alpha}_{P_{0}}\left(\mathcal{D}_{\hat{\mu}\left(x, t_{r}\right)}\right)\right)} \\
& \underset{\zeta \rightarrow 0}{=} 1-\partial_{x} \ln \theta_{0} \zeta+\left(\frac{1}{2} \partial_{x}^{2} \ln \theta_{0}+\frac{1}{2}\left(\partial_{x} \ln \theta_{0}\right)^{2}-\partial_{\underline{u}_{3}^{(2)}} \ln \theta_{0}\right) \zeta^{2}+O\left(\zeta^{3}\right), \\
& P \rightarrow P_{\infty},
\end{aligned}
$$

where $\theta_{0}=\theta\left(\underline{\Xi}_{P_{0}}-\underline{A}_{P_{0}}\left(P_{\infty}\right)+\underline{\alpha}_{P_{0}}\left(\mathcal{D}_{\hat{\hat{\mu}}\left(x, t_{r}\right)}\right)\right)$. Similarly, we can have

$$
\frac{\theta\left(\underline{\lambda}\left(P, \underline{\hat{\mu}}\left(x_{0}, t_{0, r}\right)\right)\right)}{\theta\left(\underline{\lambda}\left(P_{\infty}, \underline{\hat{\mu}}\left(x_{0}, t_{0, r}\right)\right)\right)} \underset{\zeta \rightarrow 0}{=} O(1), \quad P \rightarrow P_{\infty} .
$$

So, we give the Taylor expansion about $\psi_{1}$ as follows:

$$
\begin{aligned}
\psi_{1}\left(P, x, x_{0}, t_{r}, t_{0, r}\right) & \\
\underset{\zeta \rightarrow 0}{=} & \left(1-\partial_{x} \ln \theta_{0} \zeta+\left(\frac{1}{2} \partial_{x}^{2} \ln \theta_{0}+\frac{1}{2}\left(\partial_{x} \ln \theta_{0}\right)^{2}-\partial_{\underline{u}_{3}^{(2)}} \ln \theta_{0}\right) \zeta^{2}+O\left(\zeta^{3}\right)\right) O(1) \\
& \times \exp \left[\left(x-x_{0}\right)\left(\zeta^{-1}+q_{1} \zeta-q_{2} \zeta^{2}+O\left(\zeta^{3}\right)\right)\right] \\
\times & {\left[\left(t_{r}-t_{0, r}\right) \sum_{l=0}^{r}\left(\tilde{\beta}_{r-l} \frac{1}{\zeta^{3 l+2}}+\tilde{\alpha}_{r-l} \frac{1}{\zeta^{3 l+1}}\right)+O(\zeta)\right], \quad P \rightarrow P_{\infty} }
\end{aligned}
$$

Then it is clear that

$$
\begin{aligned}
& \sigma_{1, x}\left(x, t_{r}\right)=-\partial_{x}^{2} \ln \theta_{0}, \\
& \frac{1}{2} \sigma_{1, x x}\left(x, t_{r}\right)-\sigma_{1}\left(x, t_{r}\right) \sigma_{1, x}\left(x, t_{r}\right)+\sigma_{2, x}\left(x, t_{r}\right)=-\partial_{x} \partial_{\underline{u}_{3}^{(2)}} \ln \theta_{0} .
\end{aligned}
$$


If we set

$$
\psi_{1} \underset{\zeta \rightarrow 0}{=}\left(1+\sigma_{1}\left(x, t_{r}\right) \zeta+\sigma_{2}\left(x, t_{r}\right) \zeta^{2}+O\left(\zeta^{3}\right)\right) \exp (\Delta), \quad P \rightarrow P_{\infty}
$$

with $\Delta=\left(x-x_{0}\right)\left(\zeta^{-1}+q_{1} \zeta-q_{2} \zeta^{2}+O\left(\zeta^{3}\right)\right)+\left(t_{r}-t_{0, r}\right) \sum_{l=0}^{r}\left(\tilde{\beta}_{r-l} \frac{1}{\zeta^{3 l+2}}+\tilde{\alpha}_{r-l} \frac{1}{\zeta^{3 l+1}}\right)+O(\zeta)$, then we can show

$$
\begin{aligned}
& \psi_{1, x} \underset{\zeta \rightarrow 0}{=}\left(\sigma_{1, x} \zeta+\sigma_{2, x} \zeta^{2}+O\left(\zeta^{3}\right)\right) \exp (\Delta)+\left(\zeta^{-1}+q_{1} \zeta-q_{2} \zeta^{2}+O\left(\zeta^{3}\right)\right) \psi_{1}, \\
& \underset{\zeta \rightarrow 0}{=} \zeta^{-1} \psi_{1}+O(\zeta) \psi_{1} \\
& \psi_{1, x x} \underset{\zeta \rightarrow 0}{=}\left(\sigma_{1, x}+\left(\sigma_{2, x}+\sigma_{1, x x}\right) \zeta+\left(\sigma_{2, x x}+q_{1} \sigma_{1, x}\right) \zeta^{2}+O\left(\zeta^{3}\right)\right) \exp (\Delta) \\
& +\left(\zeta^{-1}+q_{1} \zeta-q_{2} \zeta^{2}+O\left(\zeta^{3}\right)\right) \psi_{1, x} \\
& \psi_{1, x x x} \underset{\zeta \rightarrow 0}{=}\left(3 \sigma_{1, x x}+2 \sigma_{2, x}+2 \sigma_{1, x} \zeta^{-1} O(\zeta)\right) \exp (\Delta)+\left(\zeta^{-2}+2 q_{1}-2 q_{2} \zeta+O\left(\zeta^{2}\right)\right) \psi_{1, x} \\
& \underset{\zeta \rightarrow 0}{=} 3\left(\sigma_{1, x x}+\sigma_{2, x}-\sigma_{1} \sigma_{1, x}-q_{2}\right) \psi_{1}+3\left(\sigma_{1, x}+q_{1}\right) \psi_{1, x}+\zeta^{-3} \psi_{1}+O(\zeta) \psi_{1}, \\
& P \rightarrow P_{\infty} .
\end{aligned}
$$

On the other hand, we know that

$$
\psi_{1, x x x}=\left(u_{x}\left(x, t_{r}\right)+v\left(x, t_{r}\right)+\lambda\right) \psi_{1}+2 u\left(x, t_{r}\right) \psi_{1, x} .
$$

Hence

$$
\begin{aligned}
& u\left(x, t_{r}\right)=\frac{3}{2}\left(\sigma_{1, x}+q_{1}\right), \\
& v\left(x, t_{r}\right)=3\left(\sigma_{1, x x}+\sigma_{2, x}-\sigma_{1} \sigma_{1, x}-q_{2}\right)-u_{x}\left(x, t_{r}\right) .
\end{aligned}
$$

That is just (4.40).

\section{Competing interests}

The authors declare that they have no competing interests.

\section{Authors' contributions}

All authors drafted the manuscript, read and approved the final manuscript.

\section{Author details}

${ }^{1}$ Department of Mathematics and Information Science, Zhengzhou University of Light Industry, Zhengzhou, 450002,

China. ${ }^{2}$ Department of Economics, Zhengzhou Vocational College of Economics and Trade, Zhengzhou, 450000, China.

\section{Acknowledgements}

This work was supported by the NSFC (No. 11326166) and the Doctor Foundation of Zhengzhou University of Light Industry (No. 2013BSJJ051).

Received: 11 March 2014 Accepted: 3 July 2014 Published: 23 Jul 2014

\section{References}

1. Matveev, VB, Smirnov, AO: On the Riemann theta function of a trigonal curve and solutions of the Boussinesq and KP equations. Lett. Math. Phys. 14, 25-31 (1987)

2. Brezhnev, YV: Finite-band potentials with trigonal curves. Theor. Math. Phys. 133, 1657-1662 (2002)

3. Baldwin, S, Eilbeck, JC, Gibbons, J, Ônishi, Y: Abelian functions for cyclic trigonal curves of genus 4. J. Geom. Phys. 58, 450-467 (2008) 
4. Korpel, A, Banerjee, PP: A heuristic guide to nonlinear dispersive wave equations and soliton-type solutions. Proc. IEEE 72, 1109-1130 (1984)

5. Hereman, W, Banerjee, PP, Korpel, A, Assanto, G: Exact solitary wave solutions of non-linear evolution and wave equations using a direct algebraic method. J. Phys. A, Math. Gen. 19, 607-628 (1986)

6. Yan, ZY: New families of solitons with compact support for Boussinesq-like $B(m, n)$ equations with fully nonlinear dispersion. Chaos Solitons Fractals 14, 1151-1158 (2002)

7. Fu, ZT, Liu, SK, Liu, SD, Zhao, Q: The JEFE method and periodic solutions of two kinds of nonlinear wave equations. Commun. Nonlinear Sci. Numer. Simul. 8, 67-75 (2003)

8. Lai, HL, Ma, CF: The lattice Boltzmann model for the second-order Benjamin-Ono equations. J. Stat. Mech. 4, P04011 (2010)

9. Xu, ZH, Xian, DQ, Chen, HL: New periodic solitary-wave solutions for the Benjamin Ono equation. Appl. Math. Comput. 215, 4439-4442 (2010)

10. Taghizadeh, N, Mirzazadeh, M, Farahrooz, F: Exact soliton solutions for second-order Benjamin-Ono equation. Appl. Appl. Math. 6, 384-395 (2011)

11. Date, E, Tanaka, S: Periodic multi-soliton solutions of Korteweg-de Vries equation and Toda lattice. Prog. Theor. Phys. Suppl. 59, 107-125 (1976)

12. Ma, YC, Ablowitz, MJ: The periodic cubic Schrödinger equation. Stud. Appl. Math. 65, 113-158 (1981)

13. Geng, XG, Dai, HH, Zhu, JY: Decomposition of the discrete Ablowitz-Ladik hierarchy. Stud. Appl. Math. 118, 281-312 (2007)

14. Gesztesy, F, Holden, H: A combined sine-Gordon and modified Korteweg-de Vries hierarchy and its algebro-geometric solutions. In: Differential Equations and Mathematical Physics, Birmingham, AL, pp. 133-173 (1999)

15. Geng, XG, Cao, CW: Decomposition of the $(2+1)$-dimensional Gardner equation and its quasi-periodic solutions. Nonlinearity 14, 1433-1452 (2001)

16. Dickson, R, Gesztesy, F, Unterkofler, K: Algebro-geometric solutions of the Boussinesq hierarchy. Rev. Math. Phys. 11, 823-879 (1999)

17. Geng, XG, Wu, LH, He, GL: Algebro-geometric constructions of the modified Boussinesq flows and quasi-periodic solutions. Physica D 240, 1262-1288 (2011)

18. Geng, XG, Wu, LH, He, GL: Quasi-periodic solutions of the Kaup-Kupershmidt hierarchy. J. Nonlinear Sci. 23, 527-555 (2013)

19. He, GL, Geng, XG, Wu, LH: Algebro-geometric quasi-periodic solutions to the three-wave resonant interaction hierarchy. SIAM J. Math. Anal. 46, 1348-1384 (2014)

20. Dubrovin, BA: Theta functions and nonlinear equations. Russ. Math. Surv. 36, 11-92 (1981)

21. Griffiths, P, Harris, J: Principles of Algebraic Geometry. Wiley, New York (1994)

22. Mumford, D: Tata Lectures on Theta II. Birkhäuser, Boston (1984)

10.1186/1687-1847-2014-195

Cite this article as: $\mathrm{He}$ and $\mathrm{He}$ : The application of trigonal curve theory to the second-order Benjamin-Ono hierarchy. Advances in Difference Equations 2014, 2014:195

\section{Submit your manuscript to a SpringerOpen ${ }^{\circ}$ journal and benefit from:}

- Convenient online submission

Rigorous peer review

- Immediate publication on acceptance

Open access: articles freely available online

- High visibility within the field

- Retaining the copyright to your article 